\title{
Frontal Eye Field Inactivation Diminishes Superior Colliculus Activity, But Delayed Saccadic Accumulation Governs Reaction Time Increases
}

\author{
Tyler R. Peel, ${ }^{1,4}$ Suryadeep Dash, ${ }^{1,2}$ Stephen G. Lomber, ${ }^{1,2,3,5}$ and $\bigodot_{\text {Brian D. Corneil }}^{1,2,3,5}$ \\ ${ }^{1}$ The Brain and Mind Institute, ${ }^{2}$ Department of Physiology \& Pharmacology, ${ }^{3}$ Department of Psychology, and ${ }^{4}$ Graduate Program in Neuroscience, \\ University of Western Ontario, London, Ontario N6A 5B7, Canada, and 5Robarts Research Institute, London, Ontario N6A 5K8, Canada
}

Stochastic accumulator models provide a comprehensive framework for how neural activity could produce behavior. Neural activity within the frontal eye fields (FEFs) and intermediate layers of the superior colliculus (iSC) support such models for saccade initiation by relating variations in saccade reaction time (SRT) to variations in such parameters as baseline, rate of accumulation of activity, and threshold. Here, by recording iSC activity during reversible cryogenic inactivation of the FEF in four male nonhuman primates, we causally tested which parameter(s) best explains concomitant increases in SRT. While FEF inactivation decreased all aspects of ipsilesional iSC activity, decreases in accumulation rate and threshold poorly predicted accompanying increases in SRT. Instead, SRT increases best correlated with delays in the onset of saccade-related accumulation. We conclude that FEF signals govern the onset of saccade-related accumulation within the iSC, and that the onset of accumulation is a relevant parameter for stochastic accumulation models of saccade initiation.

Key words: computational models; frontal eye fields; reversible inactivation; saccade; superior colliculus

\section{Significance Statement}

The superior colliculus (SC) and frontal eye fields (FEFs) are two of the best-studied areas in the primate brain. Surprisingly, little is known about what happens in the SC when the FEF is temporarily inactivated. Here, we show that temporary FEF inactivation decreases all aspects of functionally related activity in the SC. This combination of techniques also enabled us to relate changes in SC activity to concomitant increases in saccadic reaction time (SRT). Although stochastic accumulator models relate SRT increases to reduced rates of accumulation or increases in threshold, such changes were not observed in the SC. Instead, FEF inactivation delayed the onset of saccade-related accumulation, emphasizing the importance of this parameter in biologically plausible models of saccade initiation.

\section{Introduction}

How does the brain commit to a voluntary action? The oculomotor system that moves our line of sight provides a model system for study of this question. The primate frontal eye fields (FEFs) and intermediate layers of the superior colliculus (iSC) are two of

Received Sept. 15, 2017; revised 0ct. 20, 2017; accepted 0ct. 24, 2017.

Author contributions: T.R.P., S.G.L., and B.D.C. designed research; T.R.P. and S.D. performed research; S.G.L. contributed unpublished reagents/analytic tools; T.R.P. and B.D.C. analyzed data; T.R.P., S.D., and B.D.C. wrote the paper.

This work was supported by operating grants from the Canadian Institutes of Health Research to B.D.C. (MOPs: 93796, 123247, and 142317) and the Natural Sciences and Engineering Research Council (NSERC; RGPIN-311680). T.R.P. was supported by an Ontario Graduate Scholarship and T.R.P. and S.D, were supported by funding from an NSERC Collaborative Research and Training Experience Program grant.

The authors declare no competing financial interests.

Correspondence should be addressed to Dr. Brian D. Corneil, Robarts Research Institute, 1151 Richmond Street N, London, 0N N6A 5B7, Canada. E-mail: bcorneil@uwo.ca.

DOI:10.1523/JNEUROSCI.2664-17.2017

Copyright $\odot 2017$ the authors $\quad 0270-6474 / 17 / 3711715-16 \$ 15.00 / 0$ the most studied oculomotor structures. Within each, saccaderelated activity peaks around the time of saccade initiation (for review, see Gandhi and Katnani, 2011; Schall, 2015). Such patterns conform well to stochastic accumulator models relating neural activity to saccade initiation (Hanes and Schall, 1996; Dorris et al., 1997; Paré and Hanes, 2003; Ratcliff et al., 2003; Ding and Gold, 2012). Despite this work, simple yet fundamental questions about the oculomotor system remain unresolved. For example, what happens to iSC activity when the FEF is suddenly compromised, and how do changes in iSC activity relate to concomitant increases in saccadic reaction times (SRTs; Peel et al., 2014)? Answering these questions would not only advance basic understanding of communication within the oculomotor system, but can also test the neural implementation of stochastic accumulator models for saccade initiation. However, such answers are surprisingly hard to predict for a variety of reasons.

First, these structures are highly interconnected by monosynaptic corticotectal projections (Leichnetz et al., 1981; Komatsu 
and Suzuki, 1985) and by polysynaptic descending (e.g., through the basal ganglia or other cortical structures), ascending [e.g., via the thalamus or pulvinar (Sommer and Wurtz, 2004; Berman et al., 2009; Crapse and Sommer, 2009)], and callosal pathways (Pandya and Vignolo, 1971); the FEF and iSC also project directly to the brainstem saccadic burst generator (Raybourn and Keller, 1977; Schnyder et al., 1985; Huerta et al., 1986). Second, the functional content of signals relayed from the FEF to the superior colliculus (SC), and indeed between many different oculomotor areas, span the sensorimotor continuum (Segraves and Goldberg, 1987; Everling and Munoz, 2000; Sommer and Wurtz, 2000; Wurtz et al., 2001; Helminski and Segraves, 2003). Third, since stochastic accumulator models predict that saccades occur when activity increases above a fixed threshold, then increases in SRTs should relate to decreases in the rate at which activity accumulated and/or the baseline level of activity. However, oculomotor thresholds may not be fixed (Jantz et al., 2013) and can paradoxically decrease for longer SRTs (Heitz and Schall, 2012). Also, other parameters, such as the onset of accumulation (Pouget et al., 2011), the speed of perceptual evaluation (Shankar et al., 2011), or the period of integration (Heitz and Schall, 2012), may also affect when the oculomotor system commits to a saccade. Thus, recording iSC activity during inactivation of the FEF can not only address the contribution of the FEF to iSC activity, but can also test whether the observed profiles of iSC activity accompanying increased SRTs match those predicted by contemporary stochastic accumulator models of saccade initiation.

\section{Materials and Methods}

Subjects and surgical procedures. Four male monkeys (Macaca mulatta, monkeys M, G, D, and $\mathrm{O}$, weighing $8.7,11.1,9.8$, and $8.6 \mathrm{~kg}$ respectively) were used in these experiments. All training, surgical, and experimental procedures conformed to the policies of the Canadian Council on Animal Care and the National Institutes of Health on the care and use of laboratory animals, and were approved by the Animal Use Subcommittee of the University of Western Ontario Council on Animal Care. We monitored the monkeys' weights daily and their health was closely supervised by university veterinarians.

Each monkey underwent two surgeries to permit cryogenic inactivation of the FEF and extracellular recordings from the iSC. In the first surgery, we implanted either unilateral (right side only; monkeys $\mathrm{M}$ and $\mathrm{G}$ ) or bilateral cryoloops into the arcuate sulcus using surgical procedures previously described (Lomber et al., 1999; Peel et al., 2014). Briefly, we performed a small $2.25 \mathrm{~cm}^{2}$ craniotomy above the spur of the arcuate sulcus, and implanted two customized,

D
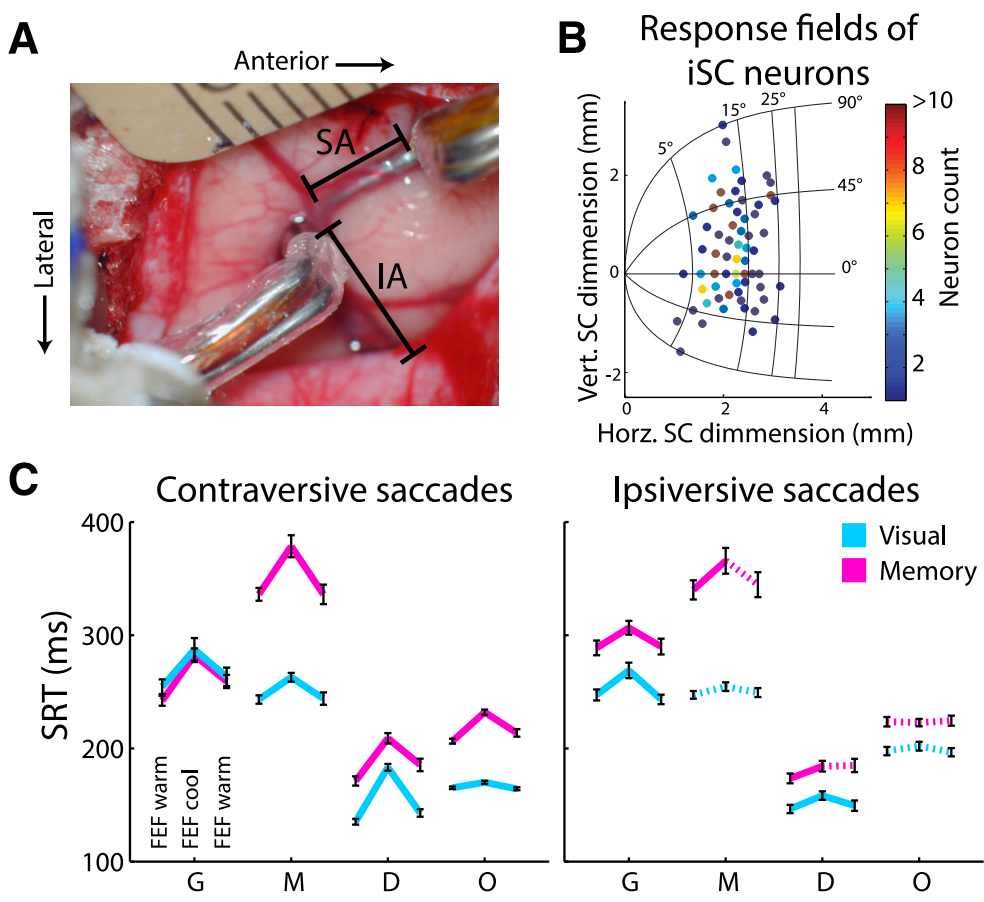

\section{Neuron responses}
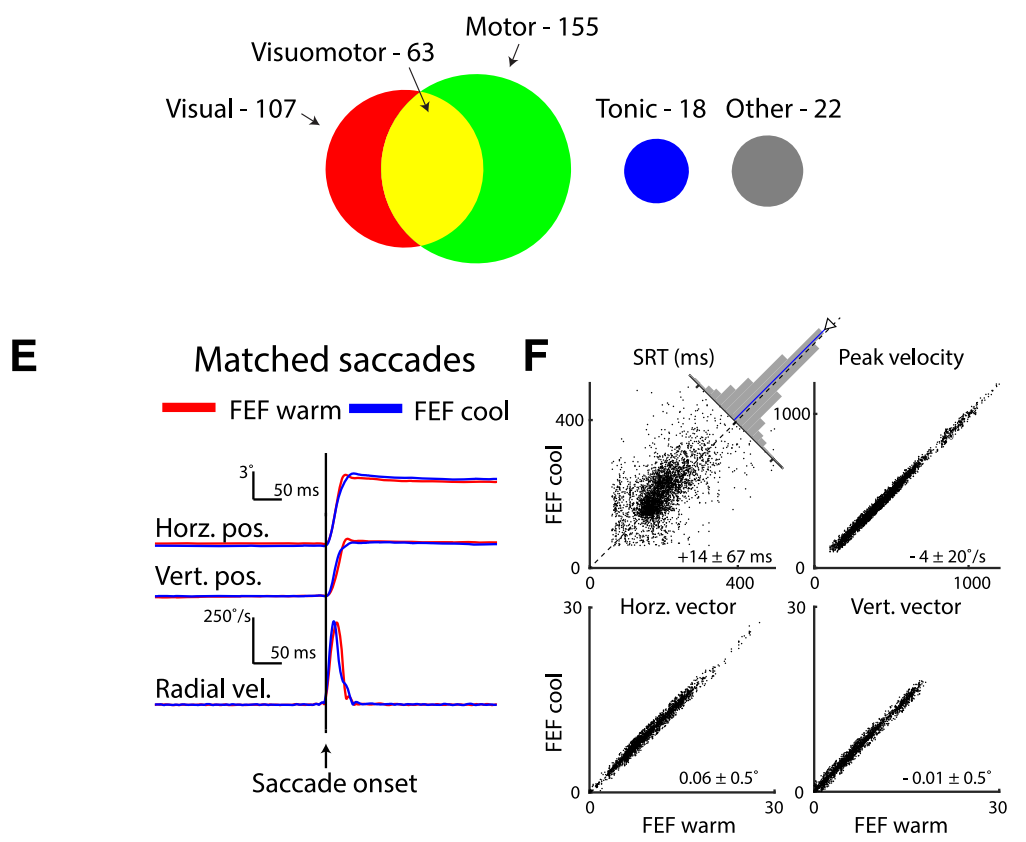

Figure 1. Materials and methods. $\boldsymbol{A}$, Cryoloops were inserted into the inferior (IA) and superior (SA) arm of the arcuate sulcus. $\boldsymbol{B}$, Response field centers for iSC neurons recorded in this study, plotted on the SC map of Hafed and Chen (2016). C, FEF inactivation increased SRTs for contraversive and occasionally ipsiversive saccades. Each line connects mean SRT ( \pm SE) across precooling, pericooling, and postcooling sessions for each of the four monkeys; solid lines indicate significant differences ( $p<0.025$, Wilcoxon signed-rank test). $\boldsymbol{D}$, Functional classification of recorded neurons (see Materials and Methods). $\boldsymbol{E}, \boldsymbol{F}$, The matched-saccade analysis compared saccades of very similar eye position and velocity profiles but different SRTs across the FEF warm or FEF cool conditions (all saccades came from the same session; see Materials and Methods). $\boldsymbol{E}$ shows one example of a saccade match; $\boldsymbol{F}$ shows characteristics of the 3762 matched saccade pairs (pooled across both ipsiversive and contraversive saccades).

stainless steel cryoloops (each 5-8 $\mathrm{mm}$ in length and $3 \mathrm{~mm}$ in depth) into the arcuate sulcus (Fig. 1A), which permitted cooling of tissue adjacent to the superior and inferior arms of the arcuate sulcus. Cryoloop temperatures of $3^{\circ} \mathrm{C}$ silence postsynaptic activity in tissue $\leq 1.5 \mathrm{~mm}$ away without influencing axonal propagation of action potentials (Lomber et al., 1999). Thermal surface imaging of the exposed tissue during surgery 
revealed that cryoloop cooling did not spread to adjacent gyri. In this manuscript, we only performed unilateral FEF inactivation and alternated the side of cooling on separate days in monkeys $\mathrm{D}$ and $\mathrm{O}$. In the second surgery, we positioned a recording chamber over a $19-\mathrm{mm}-$ diameter craniotomy to permit a surface normal approach to either iSC (Rezvani and Corneil, 2008).

Experimental procedures. Head-restrained monkeys were placed in front of a rectilinear grid of $500+$ red LEDs covering $\pm 35^{\circ}$ of the horizontal and vertical visual field. We conducted experiments in a dark, sound-attenuated room and sampled each monkey's eye position using a single, chair-mounted eye tracker at $500 \mathrm{~Hz}$ (EyeLink II, SR Research). Behavioral tasks were controlled via customized real-time LabView programs running on a PXI controller (National Instruments) at a rate of $1 \mathrm{kHz}$.

Extracellular activity was recorded on a Multichannel Acquisition Processor data acquisition system (Plexon) via tungsten microelectrodes (impedance, $0.5-3.0 \mathrm{M} \Omega$ at $1 \mathrm{kHz}$; FHC). Action potential waveforms surpassing a user-defined threshold were amplified, low-cut filtered, sorted, and stored at $40 \mathrm{kHz}$. All neurons were recorded $\sim 1 \mathrm{~mm}$ or more below the surface of the SC, in locations where electrical stimulation (300 $\mathrm{Hz}, 100 \mathrm{~ms}$, biphasic cathodal-first pulses with each phase $0.3 \mathrm{~ms}$ in duration) evoked saccades with currents $<50 \mu \mathrm{A}$. In conjunction with most recorded neurons exhibiting delay-related or saccade-related activity, recorded neurons were most likely contained with the intermediate, rather than superficial, layers of the SC, but we cannot completely rule out this possibility. We subsequently confirmed the isolation of singleunit neurons off-line throughout cooling using both sorted and unsorted action potential waveforms, and when possible ensured that the functional definition of a given neuron was maintained before and after FEF inactivation.

Upon isolating an iSC neuron, we mapped the response field for contralateral visually guided saccades. Across the 239 isolated neurons in our sample, response field centers were located at an eccentricity of $11.6 \pm$ $4.7^{\circ}$ (range, $4-25^{\circ}$ ) and at an angle relative to the horizontal axis of $12.8 \pm 30.5^{\circ}$ (range, -90 to $90^{\circ}$; Fig. $1 B$ ). After response field mapping, we attempted to collect a dataset consisting of a precooling, pericooling, and postcooling session (60 correct trials each), which required maintaining isolation for $\sim 20 \mathrm{~min}$. We largely followed a previously described procedure for cryogenic FEF inactivation (Peel et al., 2014), although to facilitate neuronal isolation through the entire dataset, we did not implement a 3 min transition between cooling sessions. Following the completion of the precooling session, chilled methanol was pumped through the lumen of the cryoloops, decreasing the cryoloop temperature. Once the cryoloop temperature was stable at $3^{\circ} \mathrm{C}$, we began the pericooling session. Upon finishing the pericooling session, we turned off the cooling pumps, which allowed the cryoloop temperature to rapidly return to normal. When the cryoloop temperature reached $35^{\circ} \mathrm{C}$, we began the postcooling session. Although saccadic behavior and iSC activity rapidly recovered after rewarming, the postcooling sessions may have contained residual effects of cooling. We controlled for this and other time-dependent factors by combining trials from precooling and postcooling sessions into the FEF warm condition. For $\sim 15 \%$ of all datasets, isolation of an iSC neuron was lost after completion of the pericooling session. We excluded postcooling trials from these sessions. Nonetheless, the effects of cooling in these sessions (based on comparing pericooling to precooling activity) were similar to the other $85 \%$ of datasets where isolation was maintained throughout the postcool session.

Behavioral tasks. Monkeys performed visually or memory-guided saccades after a delayed response period. Following a variable fixation period $(750-1000 \mathrm{~ms})$, where monkeys maintained fixation within a radius of $\pm 3^{\circ}$ of a central cue, a peripheral cue appeared in the periphery. The fixation window was purposely set to be large, as FEF inactivation can shift the average fixation position slightly (fixation position can shift by $\sim 0.5^{\circ}$ on average, although the ranges of fixation positions adopted before and during FEF inactivation still overlap; Peel et al., 2016). In 79\% of our datasets, the peripheral cue could appear either in or diametrically opposite to the center of the response field of an isolated SC neuron. In the remaining $21 \%$ of datasets, we collected data from intermixed visually or memory-guided saccades, using peripheral cues placed only in the neuron's response field. Peripheral cues were either extinguished after $250 \mathrm{~ms}$ or remained on for memory-guided or visually guided saccades, respectively. To receive a liquid reward, monkeys were required to maintain fixation throughout a fixed delay period of $1000 \mathrm{~ms}$ and to generate a saccade toward a target window (70\% of the peripheral cue's visual angle) when the central cue was extinguished. This large target window was necessary because FEF inactivation increased saccadic error, particularly for memory-guided saccades (Peel et al., 2014). The use of a fixed delay period increases anticipation of central cue disappearance, which presumably would be related to iSC activity late in the delay period.

Consistent with previous reports (Peel et al., 2014, 2016; Kunimatsu et al., 2015), large-volume unilateral FEF inactivation increased contraversive and occasionally ipsiversive saccadic reaction times (Fig. 1C) and decreased the accuracy and peak velocity of contraversive saccades. However, FEF inactivation had only a marginal effect on the monkeys' ability to perform either task, with error rates increasing at most by $14 \%$.

Neuron classification. iSC neurons were classified functionally according to a variety of response characteristics on acceptable trials (Fig. 1D). To quantify neuronal activity, we convolved spike times on individual trials with a spike-density function that mimics an excitatory postsynaptic potential (rise time of $1 \mathrm{~ms}$, decay time of $20 \mathrm{~ms}$, kernel window of 100 ms; Thompson et al., 1996). We confirmed that all our results were the same if we convolved neural activity with a $10 \mathrm{~ms}$ Gaussian.

For visual activity, we defined acceptable trials as those where the monkey maintained fixation of the central cue for the entire delay period for either visually guided or memory-guided saccades, and generated their first saccade toward the target as determined using a velocity criterion of $30 \%$ s. Then, using a Poisson analysis described previously (Hanes et al., 1995), we checked whether neurons exhibited a visually related response. Briefly, we compared the actual number of spikes within a time window to the number of spikes predicted by a Poisson distribution based on spiking activity across the entire trial. To calculate the latency of visual response within a trial, we used the time of the first burst of spikes greater than chance between 30 and $120 \mathrm{~ms}$ after cue onset; the visual latency of a given neuron was then derived by averaging the latency of detected single-trial visual responses across $\geq 8$ trials. In addition, we ensured that neurons with a visually related response had mean firing rates in the $50 \mathrm{~ms}$ interval after the average visual latency significantly greater than baseline activity integrated in the last $200 \mathrm{~ms}$ before cue onset ( $p<0.05$, Wilcoxon signed-rank test; Basso and Wurtz, 1998; McPeek and Keller, 2002). Visual activity was defined as the difference between these firing rates. We also calculated the peak magnitude of the visual response minus the baseline activity.

For delay-period and build-up activity, we applied the same criteria, but also removed any trial with anticipatory saccades (i.e., reaction time $<60 \mathrm{~ms}$ after fixation cue offset; $\sim 12 \%$ of trials). Neurons displayed delay-period activity if the mean firing rates in the last $100 \mathrm{~ms}$ of the delay period were $\geq 5$ spikes/s above baseline activity (i.e., $200 \mathrm{~ms}$ before cue onset; $p<0.05$, Wilcoxon signed-rank test; Basso and Wurtz, 1998; McPeek and Keller, 2002). The magnitude of delay-period activity was then calculated as the difference in firing rates between these intervals. Neurons with build-up activity had mean firing rates 100-200 ms before saccade onset significantly greater than the preceding $100 \mathrm{~ms}(p<0.05$, Wilcoxon signed-rank test; Anderson et al., 1998).

Finally, for saccadic activity, we used the same trials as those for the analysis of delay-period and build-up activity, but we additionally removed any trial where the monkey blinked during the first saccade $(\sim 11 \%$ of all trials in this subset). We subsequently removed any dataset with $<8$ acceptable saccades into the response field of an isolated iSC neuron either before, during, or after FEF inactivation ( $~ 9 \%$ of all datasets were removed). Neurons exhibited saccadic activity if the mean perisaccadic firing rates (defined as $8 \mathrm{~ms}$ before saccade onset to $8 \mathrm{~ms}$ before its end) were significantly greater than the last $100 \mathrm{~ms}$ of the delay period $(p<0.05$, Wilcoxon signed-rank test), and if the increase in perisaccadic activity above baseline activity in the $200 \mathrm{~ms}$ before cue onset exceeded 50 spikes/s (Munoz and Wurtz, 1995; McPeek and Keller, 2002). Saccadic activity was defined as the difference between mean perisaccadic firing rates and baseline firing rates. We also calculated the peak magnitude of saccade-related activity minus baseline activity. 
Matched saccade analysis. To examine iSC activity associated with saccade initiation and generation across FEF inactivation, the actual saccades being compared must be as similar as possible. Otherwise, any differences in saccade-related activity could be due to the generation of a saccade of different metrics, given the spatial coding of saccade metrics in the iSC, or different peak velocity, given potential relationships between the vigor of iSC activity and peak saccade velocity (Waitzman et al., 1991; Stanford et al., 1996; Katnani and Gandhi, 2012). To avoid these confounds, we matched for each neuron each FEF cool trial with one trial from a set of corresponding FEF warm trials containing similar saccade metrics and kinematics (Fig. 1E). We specified that any such matched saccades had to have horizontal and vertical displacements within $1.5^{\circ}$ and peak velocities within $50^{\circ} \mathrm{s}$. If an FEF cool trial matched with multiple FEF warm trials, we selected, with replacement, the closest match with the lowest-ranked differences in three variables (horizontal displacements, vertical displacements, and peak velocities), and occasionally by radial displacement for ties. Importantly, this ranking procedure ensured that most matches usually had differences in horizontal and vertical displacement much less than $1^{\circ}$ (mean \pm SD: $0.06 \pm 0.5^{\circ}$ and $-0.01 \pm 0.5^{\circ}$, respectively), and $<10 \%$ s in peak velocity $(-4 \pm 20 \%$; Fig. $1 F)$. Using this matching procedure, we matched $80 \%$ of FEF cool trials with a corresponding FEF warm trial recorded from the same neuron. Unless otherwise noted, we matched saccades for both metrics and kinematics for all analyses on saccade-related activity, and only analyzed saccade-related activity in neurons where we could match $\geq 5$ trials in each of the FEF warm and FEF cool conditions ( $96 \%$ of neurons with saccade-related activity met this criterion). To assess the variability inherent to this procedure with and without FEF inactivation, we also performed the same matched-saccade analysis using only FEF warm trials.

Detection of the onset of saccade-related accumulation. To investigate the neuronal correlates of SRT increases during FEF inactivation, we derived various parameters of a rise-to-threshold model (i.e., onset of accumulation relative to the go-cue, baseline, threshold, accumulation rate) from saccade-related iSC neurons. To detect the onset time of saccadic accumulation on a trial-by-trial basis, we implemented a piecewise two-piece linear regression of presaccadic iSC activity, modifying an approach described previously (Cashaback et al., 2013; Goonetilleke et al., 2015). The objective of this analysis was to find the two linear regressions that best fit the convolved iSC activity before saccade onset; the onset of saccade-related accumulation was taken as the spike time closest to the inflection point between these two linear fits. The first linear regression is based on activity from $100 \mathrm{~ms}$ before the go-cue (offset of fixation cue) to a candidate inflection point; the second linear regression is based on activity from this candidate inflection point to the peak of presaccadic activity. Candidate inflection points are tested at each millisecond within this window before peak presaccadic activity, and the onset of activity coincided with the time of the spike closest to the inflection point that minimized the summed squared error between convolved iSC activity and the two linear regressions. Note that the slope of the first linear regression was not required to be zero. Hence, it could capture any delayperiod or build-up activity the precedes saccade-related accumulation.

Of the 7524 total trials (3762 matches) matched from 193 neurons included in this analysis, we discarded a small percentage of trials (16\%) that did not reach certain criteria due to the trial-by-trial variability in saccade-related activity. Of these total trials, we removed $7 \%$ of trials with no inflection point (i.e., the minimum summed squared error occurred at the start or end of testing window), and $6 \%$ of trials where accumulation was not maintained until saccade threshold (i.e., activity decreased after the inflection point). Moreover, we also discarded 1 and $2 \%$ of total trials for when onset times occurred before the go-cue or after saccade threshold, respectively. Because we obtained identical results with or without removing a small fraction of trials with onsets before the go-cue, this suggests that anticipation of the go-cue did not influence our results. These criteria left us with a total of 5420 trials from 2710 pairs of matched trials (2083 pairs from ipsilesional iSCs, and 627 pairs from contralesional iSCs). Importantly, the $r^{2}$ values of $0.63 \pm 0.24$ (mean \pm SD) produced by this two-piece linear regression were substantially larger than those arising from a single linear fit over the same time range
$(0.34 \pm 0.21)$. Further, the $r^{2}$ values changed by $<2 \%$ during FEF inactivation $(0.63 \pm 0.25$ for FEF warm trials to $0.61 \pm 0.25$ for FEF cool trials). To ensure that the first linear regression sufficiently captured delay-period or build-up activity before the inflection point, we also performed similar analyses using larger window sizes starting before gocue $(200,350$, and $500 \mathrm{~ms}$ ) or convolution functions (e.g., a $10 \mathrm{~ms}$ Gaussian). We also tried fitting the activity before the inflection point with a quadratic function. All of these alternative analyses produced robust $r^{2}$ values of $>0.59$ and had similarly detected onset of accumulation values (median difference $<1 \mathrm{~ms}$ across all matched trials) compared with the two-piece piecewise linear-regression analysis. Although $r^{2}$ values did significantly increase with the quadratic fit (as expected given the use of an additional term), we deemed that fitting the first portion of the data with a quadratic curve was justified only when this increased the $r^{2}$ value by $10 \%$ and significantly reduced the residuals ( $F$ test with $p<0.01$; Nagy and Corneil, 2010). Using such criteria, a quadratic fit was deemed necessary on only $15 \%$ of all matched trials, and changed onset times by only $6 \pm 27 \mathrm{~ms}$ (mean $\pm \mathrm{SD}$ ) across all matched trials. We also implemented an alternative Poisson-based analysis of burst onset (Hanes et al., 1995) to verify our main results. For this alternative Poisson-based analysis, we only included trials where we could detect a burst of spikes after the go-cue and before saccade onset, with the onset time coinciding with the burst of spikes nearest to saccade onset. We obtained similar results using this alternative onset detection method (see Results).

Determining accumulation rate, baseline, and threshold. We also derived the accumulation rate, baseline, and threshold from saccaderelated iSC neurons. For the accumulation rate, we simply took the slope of the second linear regression running between the inflection point and the peak presaccadic activity. Because this calculation of accumulation rate hinges on this calculation of onset time, we also derived the accumulation rate in another way by finding the slope of the line when activity crossed the 30 and $70 \%$ points of peak presaccadic activity. This latter calculation of accumulation rate is independent of the two-piece linear regression, but yielded similar results. For baseline activity, we took the average level of activity in the $100 \mathrm{~ms}$ window before the offset of the fixation cue. For threshold activity, we calculated the activity $8-18 \mathrm{~ms}$ before saccade onset (Jantz et al., 2013; Johnston et al., 2014), which is based on the minimum amount of time for the iSC to influence the brainstem circuits regulating saccade onset (Miyashita and Hikosaka, 1996).

Finally, we conducted two further analyses to examine how combinations of the changes in the parameters of SC saccade-related activity related to SRT differences during FEF inactivation. First, we calculated the time-to-reach-threshold as the difference in the threshold and baseline activities divided by the accumulation rate, and directly related this combined measure to SRT differences using similar millisecond units. Second, because these parameters may covary, we examined the change in each parameter [onset of accumulation $(\Delta O)$, baseline $(\Delta B)$, threshold $(\Delta T)$, accumulation rate $(\Delta A)]$ in isolation by regressing such changes against the timing residuals $(\varepsilon)$ of a multiple linear regression consisting of all other parameters (Hewitt et al., 2015). This approach removed the variability associated with interactions among the other parameters, so that we could examine how well a single parameter directly contributed to SRT differences. For example, to evaluate $\Delta O$ independently of $\Delta B$, $\Delta T$, and $\Delta A$, we used the following Equation 1 to compute the $\varepsilon$ term, which represents the remaining temporal variability between $\Delta S R T$ and model parameters after everything but $\Delta O$ is removed: $\varepsilon(x)=\Delta S R T-$ $\alpha_{1}-\beta_{B} \Delta B(x)-\beta_{T} \Delta T(x)-\beta_{A} \Delta A(x)$.

After calculating the timing residuals, we then used a linear regression to fit the final parameter $(\Delta O)$ against the $\varepsilon(x)$ term as shown in the following Equation 2: $r(x)=\varepsilon(x)-\alpha_{2}-\beta_{o} \Delta O(x)$.

The goodness-of-fit $\left(r^{2}\right)$ measure taken from the residuals $r(x)$ of this linear regression represents how well changes in onset of accumulation explains SRT differences across matched trials, after removing any variability associated with interactions among the other parameters.

Experimental design and statistical analysis. To quantify the effects of FEF inactivation on visual and delay-period iSC activity, we performed two-sample Wilcoxon rank-sum tests to find statistical differences within individual neurons at $p<0.05$, and we used paired Wilcoxon signed- 
rank tests to uncover statistical differences across the neuronal population at $p<0.05$. Because we only analyzed saccadic activity from paired trials having similar saccade metrics and kinematics, we performed paired Wilcoxon signed-rank tests on both individual neurons and a population of neurons to ensure that differences in saccadic activity reached significance at $p<$ best to SRT increases in more 0.05 . We also correlated changes in saccade-related activity during FEF inactivation to concomitant SRT increases. To do this, we performed a regression analysis where we fitted a linear regression to examine how changes in iSC activity predicted SRT differences across the neuron population or all matched trials. We interpreted a significant correlation if the probability associated with the $F$ statistic (the mean regression sum of squares divided by the mean error sum of squares) was $<0.05$.

\section{Results}

We recorded activity from either the ipsilesional or contralesional iSC before, during, and after large-volume unilateral cryogenic FEF inactivation while monkeys performed delayed visually or memory-guided saccades to cues placed in or opposite to the center of the neuron's response field (see Materials and Methods; Fig. 1). We only cooled the cryoloop in the inferior arm of the arcuate sulcus, which provided an estimated volume of inactivation of $90 \mathrm{~mm}^{3}$ in the anterior bank of the arcuate sulcus. Previously, we showed that cooling only the inferior arm cryoloop produced behavioral deficits $\sim 70 \%$ of the magnitude produced by cooling both loops together (Peel et al., 2014).

The delayed nature of the behavioral tasks used here, requiring the animals to temporarily withhold a saccade to a persistent or remembered peripheral visual cue until the offset of the central fixation point, provides the opportunity to quantify the effects of FEF inactivation on different aspects of functionally defined iSC activity. Accordingly, in the first half of the results, we describe the effects of FEF inactivation on iSC activity during visual, delayperiod, build-up, and saccade-related epochs. In the second half of the results, we focus on how changes in presaccade activity in the iSC during FEF inactivation relate to the parameters derived from a stochastic accumulator model. While such models have not customarily been applied to delayed saccade tasks, they provide a framework for determining whether changes in baseline, rate of rise, threshold, or onset of accumulation best predicted the associated increases in SRT.

We recorded 239 neurons (178 ipsilesional, 61 contralesional to FEF inactivation) from the caudal iSC, maintaining isolation before and during FEF inactivation, and usually (85\%) after rewarming. Of these neurons, 107 (45\%) exhibited visual activity, 147 (62\%) had delay-period activity, 60 (25\%) had build-up activity, 155 (65\%) had saccade-related activity, and 22 neurons (9\%) did not meet any of our classification criteria. Consistent with previous work (Peel et al., 2014, 2016), unilateral FEF inactivation increased SRT toward contraversive cues and usually toward ipsiversive cues. Because FEF inactivation also impaired the accuracy and peak velocity of contraversive saccades, we performed a matched-saccade analysis to ensure analysis of saccaderelated iSC activity during the production of equivalent saccades. Such matching is crucial to avoid confounds related to the generation of a different saccade or to differing degrees of saccaderelated drive onto the brainstem burst generator (Yoshida et al., 1999).

\section{Responses of iSC neurons before FEF inactivation}

Our sample of iSC neurons exhibited visual, delay-period, and saccade-related activity, the latencies and magnitudes of which concurred well with previous reports of iSC activity (Munoz and Wurtz, 1995; Basso and Wurtz, 1998; McPeek and Keller, 2002).
Before FEF inactivation, neurons with visual activity had response latencies of $56 \pm 9 \mathrm{~ms}$ (mean $\pm \mathrm{SD}$; range, 42-99 ms), firing rates of $77 \pm 39$ spikes/s, and peak magnitudes of $106 \pm 54$ spikes/s. Eighty percent of these 107 visual neurons also exhibited delay-period (71\%), build-up (11\%), and/or saccade-related activity (59\%). Based on these results and our recording approach (see Materials and Methods), we surmise that most visual neurons resided in the intermediate rather superficial layers of the SC. However, the small subset of visual-only neurons (21 total, 14 from the ipsilesional iSC) may have been located in the superficial SC. For neurons exhibiting saccade-related activity, we found firing rates of $160 \pm 86$ spikes/s and peak magnitudes of $225 \pm$ 108 spikes/s during visually guided saccades, and corresponding activities of $124 \pm 60$ and $175 \pm 71$ spikes/s during memoryguided saccades. Eighty-three percent of 155 saccade-related neurons also exhibited visual (41\%), delay-period (68\%), and/or build-up (28\%) activity.

We now turn to the effects of FEF inactivation on iSC activity. Where possible, we controlled for time-dependent factors by combining precooling and postcooling trials into the FEF warm condition, and compared this to trials when the FEF was inactivated (the FEF cool condition). Equivalent results were obtained if we excluded the $15 \%$ of our sample where we were not able to record postcooling data.

\section{FEF inactivation reduced but did not delay ipsilateral iSC visual responses}

We first examined the effect of FEF inactivation on the magnitude and timing of visual responses in the iSC. Figure $2 \mathrm{~A}$ shows an example neuron recorded from the ipsilesional iSC before, during, and after FEF inactivation. This neuron exhibited delayperiod activity, since it also remained active at a lower rate when the cue remained on, and also exhibited saccade-related activity (data not shown). In the FEF warm condition, visual activity commenced $45 \pm 6 \mathrm{~ms}$ after cue onset (determined by a Poisson burst analysis; see Materials and Methods) and averaged $71 \pm 23$ spikes/s for the subsequent $50 \mathrm{~ms}$. FEF inactivation did not alter the visual burst onset latency ( 45 to $47 \mathrm{~ms}, p=0.99, z=-0.01$, Wilcoxon rank sum test), but decreased visual activity from 71 to 52 spikes/s ( $p=0.06, z=1.89$, Wilcoxon rank sum test).

Across our sample, FEF inactivation consistently decreased visual activity in the ipsilesional $(p<0.01, z=-2.95$, Wilcoxon signed-rank test, $\sim 3 \pm 2 \%$ decrease, mean \pm SE, in all 89 neurons, and $\sim 24 \pm 3 \%$ decrease in the 21 neurons exhibiting significantly decreased visual activity), but not contralesional iSC ( $3 \pm 4 \%$ increase; $p=0.62, z=0.50$, Wilcoxon signed-rank test; Fig. 2). A statistical comparison of changes in the magnitude of the visual response across the ipsilesional and contralesional iSC did not reach significance $(p=0.07, z=1.79$, Wilcoxon rank sum test), perhaps because of the small sample size of contralesional iSC neurons. FEF inactivation did not alter visual response latencies across either ipsilesional (increase and SE of $<1$ $\mathrm{ms}, p=0.93, z=0.09$, Wilcoxon signed-rank test) or contralesional iSC neurons (increase and SE of $<1 \mathrm{~ms}, p=0.84, z=$ 0.20 , Wilcoxon signed-rank test; Fig. $2 C$ ). When present, FEF inactivation also did not alter activity in the $200 \mathrm{~ms}$ preceding cue onset in either the ipsilesional $(13 \pm 10 \%$ increase, $p=0.87, z=$ 0.16 , Wilcoxon signed-rank test) or contralesional $(2 \pm 24 \%$ decrease, $p=0.19, z=1.36$, Wilcoxon signed-rank test) iSC across our sample, nor in the subset of 21 ipsilesional iSC neurons ( $7 \pm 14 \%$ decrease, $p=0.78, z=0.28$, Wilcoxon signed-rank test) exhibiting significantly reduced visual activity. 
A

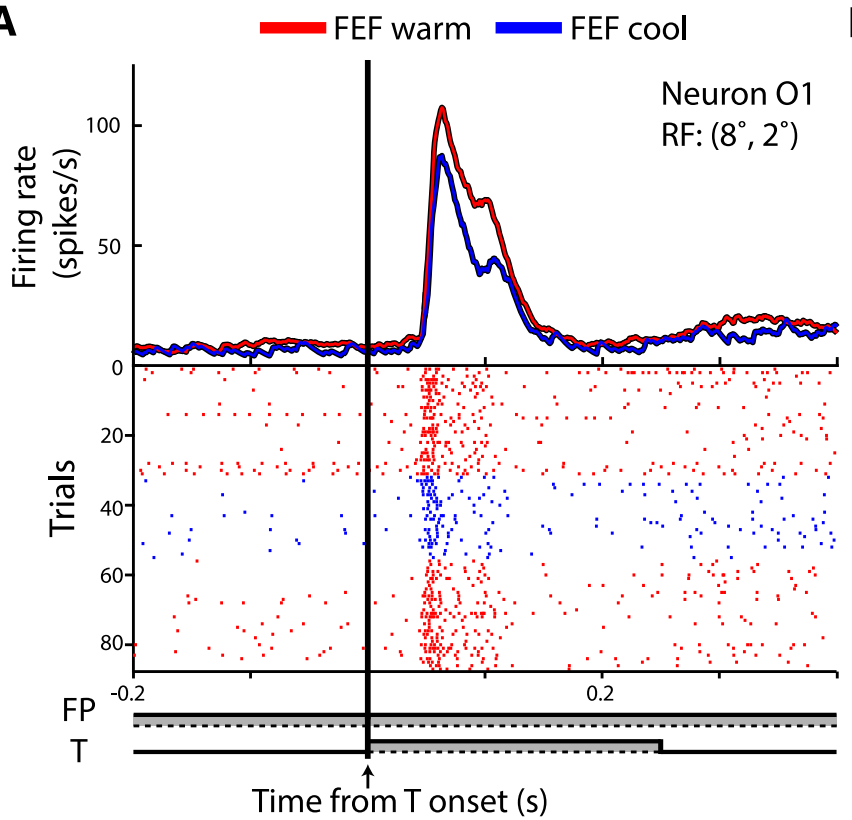

B

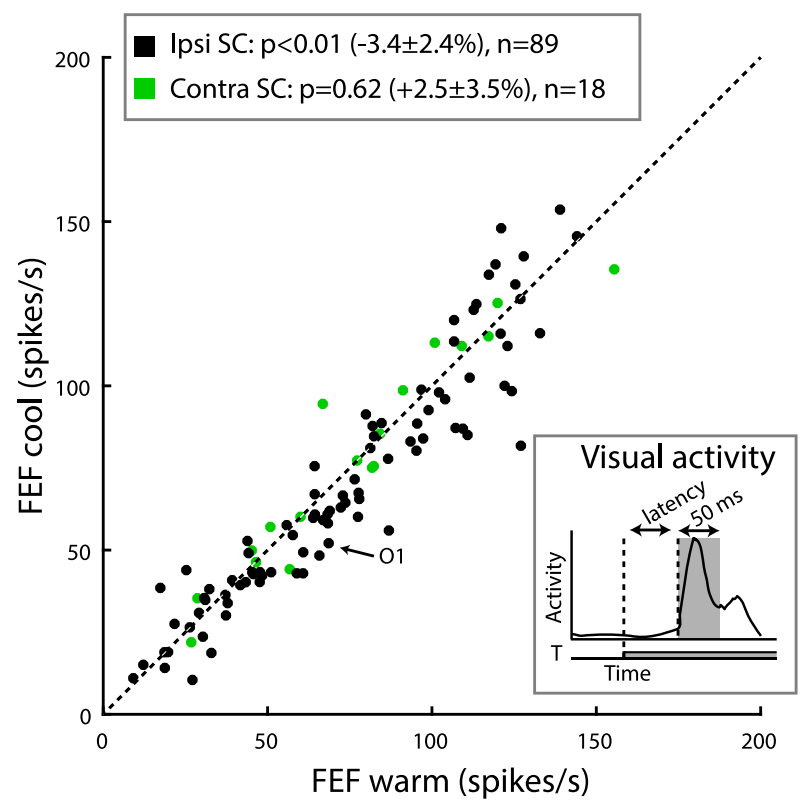

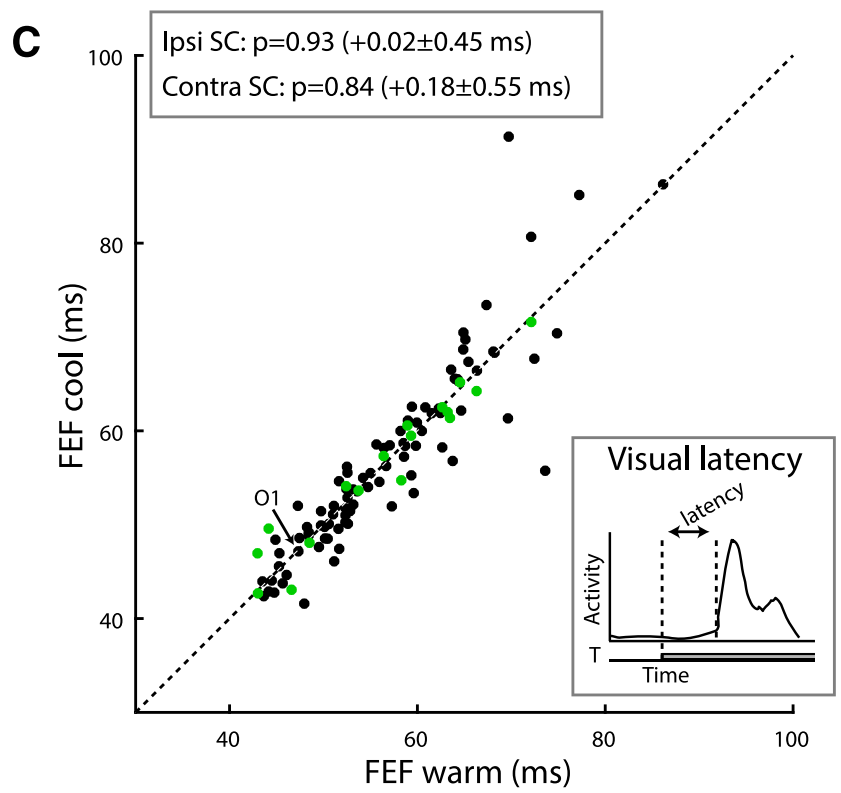

D

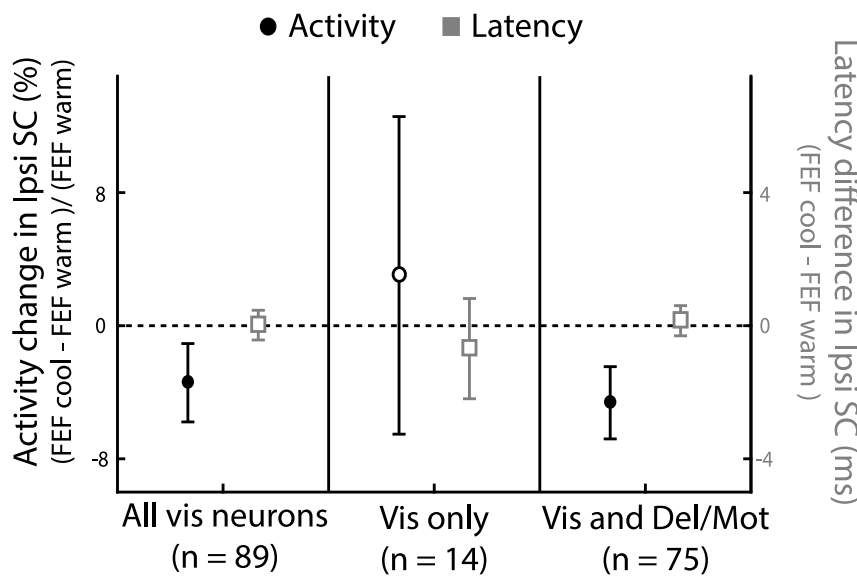

Figure 2. FEF inactivation decreased visual activity of ipsilesional iSC neurons. $A$, Spike rasters (bottom) and mean spike-density functions (top) showing reduced visual response to peripheral cue onset on ipsilesional iSC neuron 01 with FEF inactivation (FP, fixation point; T, target). $B, C$, FEF inactivation decreased activity in the 50 ms interval following the start of the visual response in the ipsilesional (black circles) but not contralesional (green circles) iSC (B), without altering visual response latency in either iSC ( $\boldsymbol{C}$; line represents line of unity; $\boldsymbol{P}$ value shows results of Wilcoxon signed-rank test). $D$, FEF inactivation decreased visual responses of neurons exhibiting other functional responses (left axis, black circles, percentage change $\pm \mathrm{SE}$ ), but did not alter visual response latency (right axis, gray squares; difference \pm SE). Filled symbols represent significant effects using Wilcoxon signed-rank test $(p<0.05)$.

The effect of FEF inactivation on the magnitude of visual responses depended on the functional classification of the recording neuron, being more pronounced in ipsilesional iSC neurons that also displayed delay-related and/or saccade-related activity $(5 \pm 2 \%$ decrease, $p<0.01, z=-2.65$, Wilcoxon signed-rank test, $n=75$ ), compared with the putative superficial SC neurons, which only exhibited a visual response $(3 \pm 10 \%$ increase, $n=14$; Fig. 2D). Although FEF inactivation increased the response magnitude of visual-only neurons, this increase was not significant ( $p=0.15, z=-1.48$, Wilcoxon signed-rank test) given a small sample size and a high degree of variability between neurons. In terms of response latency, and in contrast to the effects of FEF inactivation on response magnitude, we did not observe any influence of FEF inactivation on visual latency of SC neurons when subdivided into different functional classifications (all differences, $<1 \mathrm{~ms} ; p=0.98$ and $0.77, z=-0.03$ and 0.28 , Wilcoxon signed-rank tests on visual-only and multiple-response neurons, respectively; Fig. 2D).

\section{FEF inactivation decreased delay-period activity in ipsilesional iSC neurons}

FEF inactivation reduced delay-period activity in ipsilesional iSC neurons both in the presence (visually guided saccades) and absence (memory-guided saccades) of peripheral cues. This result is shown for two representative ipsilesional iSC neurons (Fig. $3 A, B)$. In the memory-guided saccade task, the neuron shown in Figure $3 A$ displayed modest delay-period activity of $13 \pm 4$ spikes/s in the last $100 \mathrm{~ms}$ period before fixation cue offset, which 
A

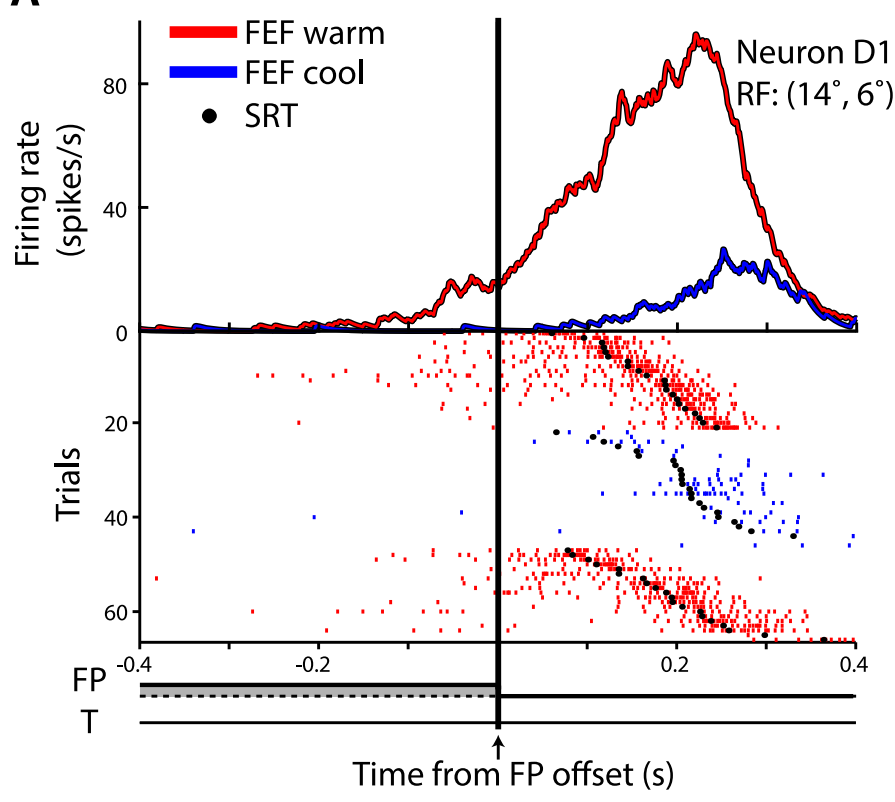

C

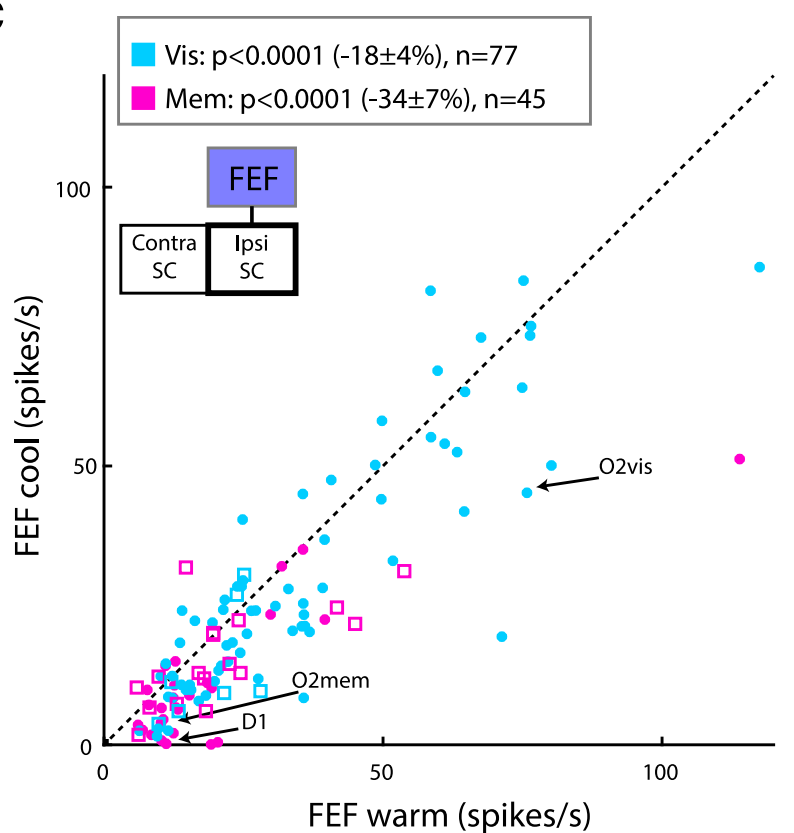

B

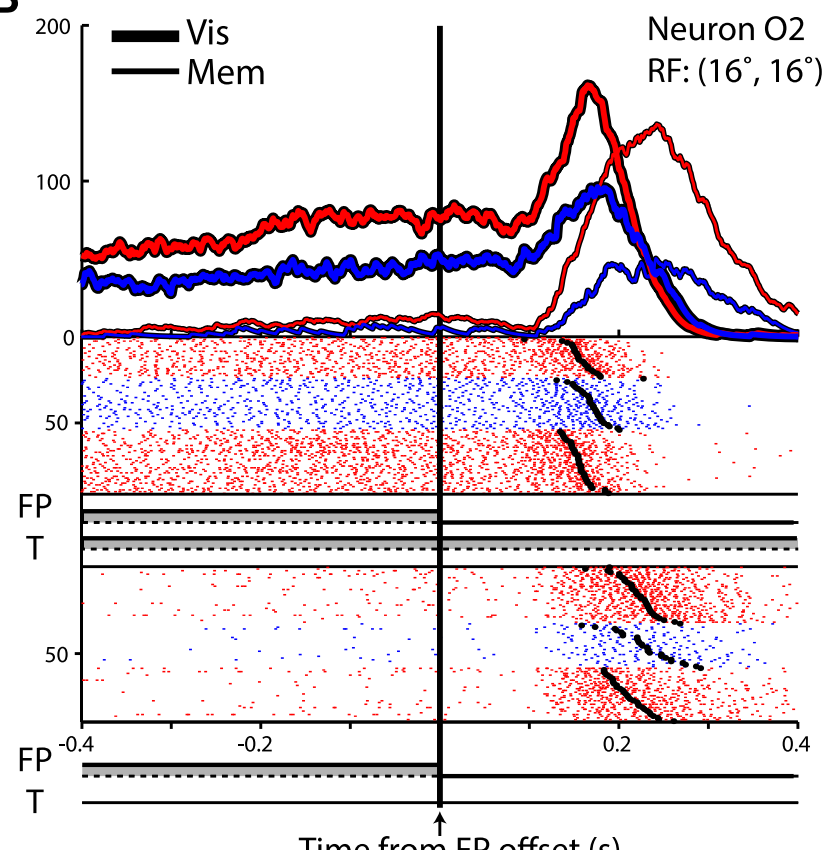

D

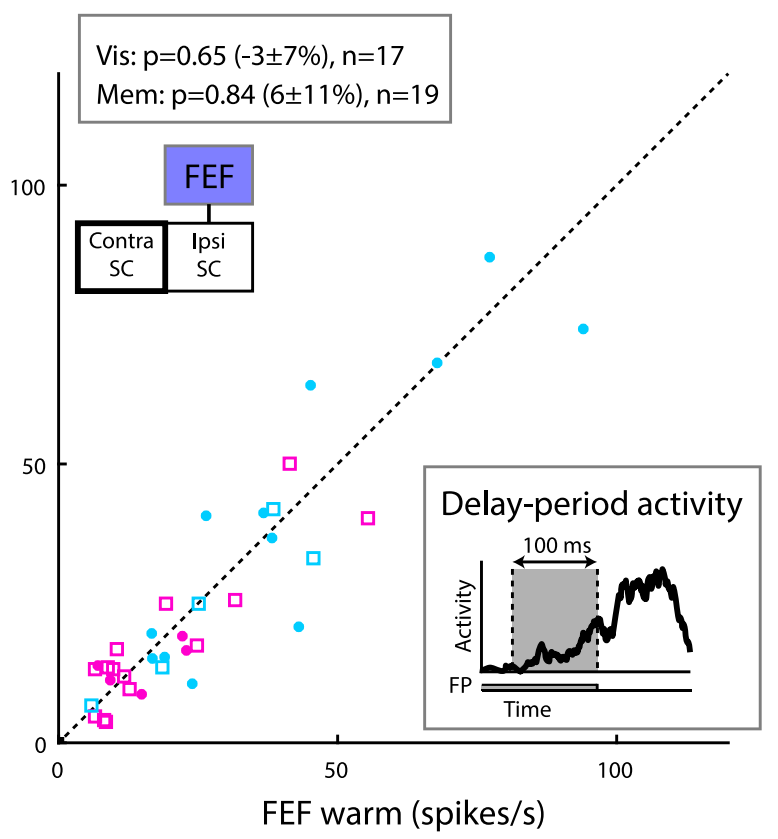

Figure 3. FEF inactivation decreased delay-period activity of ipsilesional iSC neurons. $\boldsymbol{A}, \boldsymbol{B}$, FEF inactivation nearly abolished modest delay-period activity in ipsilesional iSC neuron D1 during a memory-guided saccade $(\boldsymbol{A})$, and reduced delay-period activity in ipsilesional iSC neuron 02 in both the visually and memory-guided tasks $(\boldsymbol{B})$. $\boldsymbol{C}, \boldsymbol{D}$, FEF inactivation consistently decreased delay-period activity in the last 100 ms before peripheral cue offset for both visually and memory-guided tasks in ipsilesional (C) but not contralesional (D) iSC neurons (squares or circles denote neurons also displaying build-up activity or not, respectively; same general format as Fig. 2).

ramped up until saccade onset. FEF inactivation effectively abolished this delay-period activity (decreasing from 13 to 0.2 spikes/s; $p<0.0001, z=4.90$, Wilcoxon rank sum test). The visuomotor neuron shown in Figure $3 B$ was recorded during interleaved visually and memory-guided saccades, and exhibited far greater delay-period activity when the cue was present $(73 \pm 6$ spikes/s) than absent (12 \pm 2 spikes/s). FEF inactivation decreased delay-period activity both when cues were present $(73$ to 45 spikes/s; $p<0.0001, z=4.41$, Wilcoxon rank sum test) or absent ( 12 to 5 spikes/s; $p<0.05, z=2.23$, Wilcoxon rank sum test). Across our sample, FEF inactivation robustly reduced delay-period activity in both saccade tasks in the ipsilesional iSC
(Fig. 3C; visually guided, cyan, $p<0.0001, z=-4.07,31 \%$ of 77 neurons exhibited significant decreases; memory-guided, magenta, $p<0.0001, z=-4.67$, Wilcoxon signed rank test, $40 \%$ of 45 neurons exhibited significant decreases). The effects of FEF inactivation were the same on ipsilesional neurons with (squares) or without (circles) build-up activity before saccade onset, but proportionally larger during the memory-guided $34 \pm 7 \%$ decrease in all 45 neurons, or $68 \pm 6 \%$ decrease in the 18 neurons with significant decreases) versus visually guided (18 $\pm 4 \%$ decrease in all 77 neurons, or $51 \pm 4 \%$ decrease in the 24 neurons with significant decreases) saccade task. In contrast, we observed no effect of FEF inactivation on delay-period activity in our sam- 
ple of contralesional iSC neurons (Fig. $3 D ; p=0.84$ and $0.65, z=$ -0.20 and -0.45 , Wilcoxon signed-rank tests for memoryguided and visually guided, respectively). The lack of changes on contralesional iSC neurons during FEF inactivation is also supported by FEF inactivation producing larger decreases of delayperiod activity in the ipsilesional compared with the contralesional iSC $(p<0.01$ and $0.1, z=2.76$ and 1.37 , Wilcoxon rank sum tests for memory-guided and visually guided, respectively).

As previously mentioned, FEF inactivation had a stronger effect on visual neurons that also exhibited delay-related or saccade-related activity. We found a similar pattern for the 113 ipsilesional iSC neurons with delay-period activity: 40 of 42 neurons exhibiting significantly reduced delay-period activity during FEF inactivation also exhibited either visual (62\%), build-up $(21 \%)$, or saccade-related activity $(71 \%)$. Likewise, FEF inactivation consistently decreased visual ( $p<0.001, z=3.57$, Wilcoxon signed-rank test) and saccade-related activity before both visually $(p<0.001, z=-3.59$, Wilcoxon signed rank test $)$ and memoryguided saccades ( $p<0.05, z=-2.29$, Wilcoxon signed-rank test) in this subset of 40 neurons.

\section{FEF inactivation reduced saccade-related activity of ipsilesional iSC neurons}

Next, we examined the FEF's contribution to saccade-related activity in the ipsilesional and contralesional iSC. For this analysis, saccades generated during FEF inactivation must be matched as closely as possible for metrics and velocity, otherwise any changes in saccade-related activity could simply arise from the generation of a different saccade. We matched saccades within $1.5^{\circ}$ of horizontal and vertical displacement and within $50 \%$ of radial peak velocity, although we typically found matches well within these limits using a ranking procedure (see Materials and Methods; Fig. $1 F)$. Despite the generation of effectively equivalent saccades, FEF inactivation decreased ipsilesional saccade-related activity in ipsilesional iSC neurons. This result is shown for one neuron in Figure $4 A$, where activity for visually guided saccades decreased significantly from $301 \pm 85$ to $263 \pm 52$ spikes/s $(p<0.001, z=$ 3.61, Wilcoxon signed-rank test). Across our sample, FEF inactivation consistently decreased saccade-related activity for ipsilesional iSC neurons ( $\geq 5$ matches) for visually or memory-guided saccades (each $p<0.0001, z=-5.37$ and -5.88 , Wilcoxon signedrank tests, respectively; Fig. $4 B$ ). The proportional decrease caused by FEF inactivation was greater for memory-guided versus visually guided saccades, both in terms of how much activity decreased ( $20 \pm 3$ and $11 \pm 2 \%$ decrease, respectively) and in the proportion of neurons exhibiting significantly changed activity (69\% of 59 neurons and $58 \%$ of 77 neurons, respectively).

Another way of analyzing the effects of FEF inactivation on saccade-related iSC activity is to directly compare activity for all matched saccades, pooled across all recorded neurons. The result of this analysis is shown in Figure $4 C$ (top row), where each point represents saccade-related activity for a matched saccade generated during the FEF cool versus FEF warm condition. The clustering of points below the line of unity in the top row of Figure $4 C$, as well as the rightward skew of the blue histograms, reinforces how FEF inactivation decreases ipsilesional iSC saccade-related activity. As a control, we performed a similar saccade-matching procedure using FEF warm trials. While there is considerable scatter in this analysis around the line of unity (Fig. 4C, bottom row), the resulting distributions of the red histograms were not skewed away from zero.
Finally, while FEF inactivation significantly decreased saccaderelated activity in some contralesional iSC neurons (20 and 29\% of neurons for visually and memory-guided, respectively), such effects were less consistent across our sample (Fig. $4 D ; 3 \pm 5 \%$ decrease for visually guided, $p=0.71, z=-0.37 ; 11 \pm 5 \%$ decrease for memory-guided, $p<0.05, z=-2.14$, Wilcoxon signed-rank tests). Further, FEF inactivation decreased saccade-related activity significantly more for ipsilesional versus contralesional iSC neurons during both the memory-guided saccade task $(p<0.05$, $z=-2.35$, Wilcoxon rank sum test), but not the visually guided saccade task ( $p=0.07, z=-1.82$, Wilcoxon rank sum test).

\section{Summary of effects of FEF inactivation on iSC activity}

The results up to this point have focused on how FEF inactivation decreased visual, delay-period, and saccade-related activity in the downstream iSC, particularly in the ipsilesional side. In Figure 5, we summarize the impact of FEF inactivation on all aspects of functionally defined activity in the ipsilesional iSC by presenting average spike-density functions across our sample, separated where appropriate for visually and memory-guided saccades. Presenting the data in this way emphasizes the greater impact of FEF inactivation on memory-guided saccades, which is consistent with previous behavioral (Peel et al., 2014) and neurophysiological (Sommer and Wurtz, 2000) findings of a larger contribution of FEF to working memory, and also makes comparisons with other studies easier (Koval et al., 2011; Johnston et al., 2014).

\section{Delays in the onset of iSC saccade-related accumulation predicted bilateral SRT increases}

One of the intriguing features in Figures 4 and 5 is that saccaderelated activity decreases during FEF inactivation, despite concomitant increases in SRT. Were SRT increases to be related to saccade threshold, then SC activity should have increased upon FEF inactivation. To reconcile these observations, we next examined how FEF inactivation altered the parameters of iSC presaccadic activity derived from a modified stochastic accumulator model (i.e., baseline and threshold activity, onset and rate of accumulation; see Materials and Methods), and determined which of these parameters best predicted the accompanying SRT increases. Within this model, increased SRTs could arise from one or some combination of decreases in baseline activity or rate of accumulation, or from increases in onset of accumulation or saccade threshold.

We first focus on the onset of accumulation. To find the onset of accumulation for each trial, we used a piecewise two-piece linear-regression method (Fig. 6A; see Materials and Methods). This method systematically tests within a window for the inflection point between two linear regressions (one for baseline and one for the rise in activity before saccade onset), with the onset of accumulation coinciding with the inflection point that minimizes the sum-of-square values for the two linear regressions against the convolved spike-density function.

The influence of FEF inactivation on the onset of accumulation (black ticks) and SRT (black circles) is shown for a representative neuron in Figure $6 B$, showing single-trial activity for one pair of matched saccades (Fig. 6B, top row; inset shows the position and velocity profiles for this match), and across all matches recorded from this neuron (Fig. $6 B$, bottom). Across all matched trials for this neuron, FEF inactivation increased the average onset of accumulation from $142 \pm 16$ to $162 \pm 22 \mathrm{~ms}$ after fixation cue offset ( $p<0.0001, z=-3.91$, Wilcoxon signed-rank test) and significantly increased the average SRT of matched memoryguided saccades from $207 \pm 20$ to $238 \pm 29 \mathrm{~ms}(p<0.0001$, 

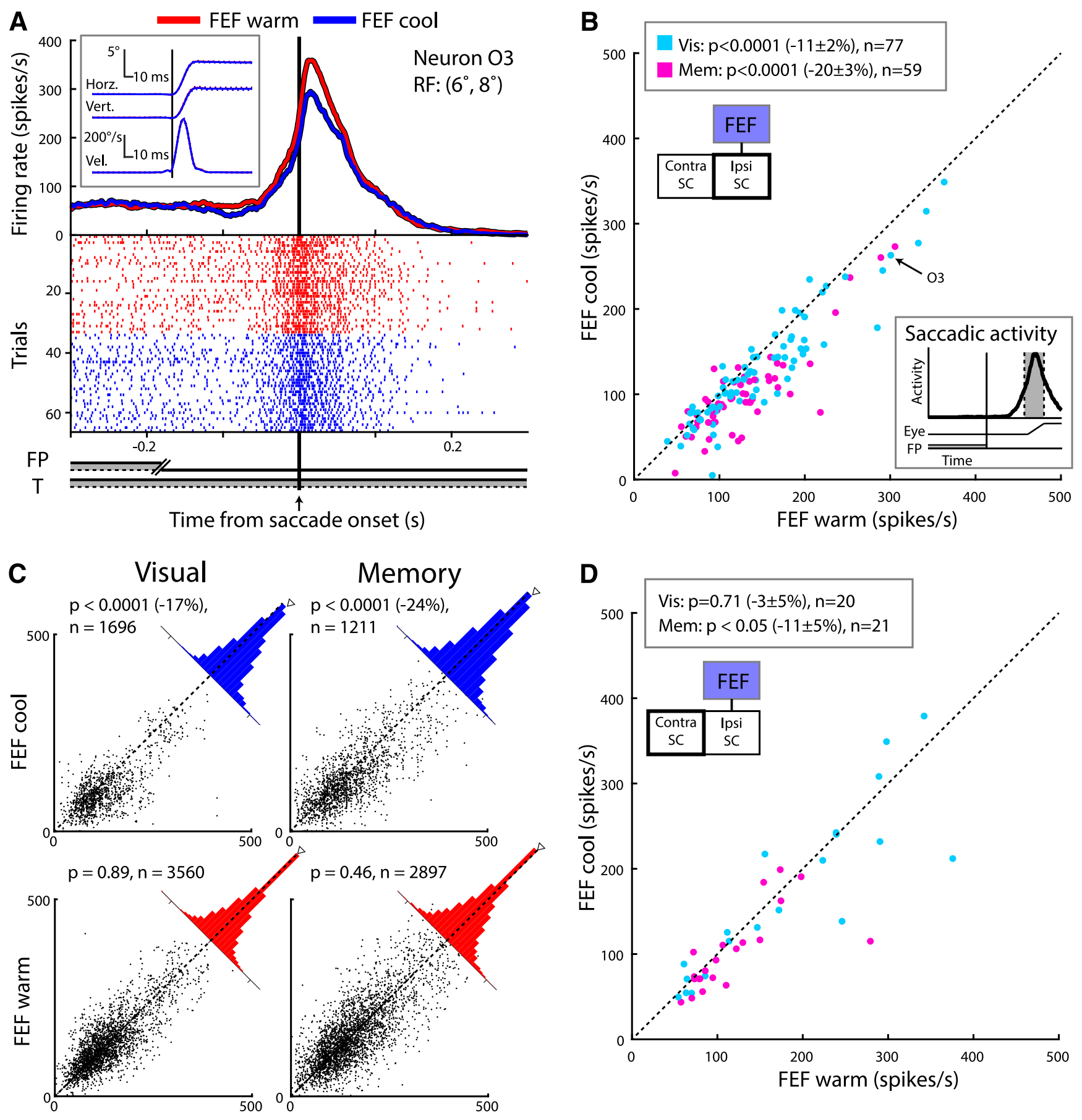

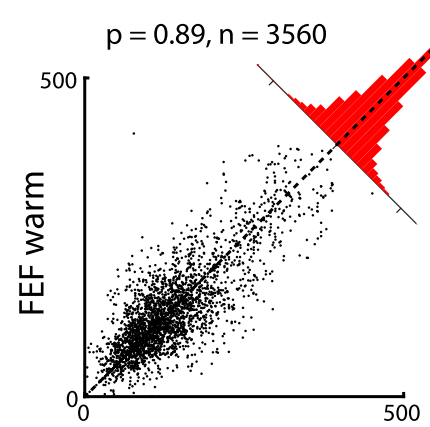

FEF warm

FEF warm

Figure 4. FEF inactivation decreased saccade-related activity of ipsilesional iSC neurons. A, FEF inactivation decreased saccade-related activity in ipsilesional iSC neuron 03 (inset shows position and velocity profiles for matched visually guided saccades). $\boldsymbol{B}$, FEF inactivation consistently decreased saccade-related activity ( $8 \mathrm{~ms}$ before saccade onset to $8 \mathrm{~ms}$ before saccade offset; see schematic) for ipsilesional iSC for both visually and memory-guided saccades (same format as Fig. $2 B$; neurons included only if they had $\geq 5$ matched saccades). C, Direct comparison of saccade-related activity for all matched contralesional saccades. FEF inactivation generally decreased saccade-related activity (top row, blue). As a control, we also matched saccades from FEF warm trials (bottom row, red) and did not find consistently decreased saccade-related activity. D, FEF inactivation did not consistently influence saccade-related activity in the contralesional isC.

$z=-4.39$, Wilcoxon signed-rank test). Thus, in this example, FEF inactivation delayed onset of iSC accumulation and increased SRT.

Across our sample, FEF inactivation delayed the onset of iSC accumulation in the ipsilesional iSC for memory-guided (Fig. $6 C$; $12 \pm 3$ ms increase, $p<0.001, z=3.53$, Wilcoxon signed-rank test; onsets from individual trials were averaged within neurons for this analysis) saccades, but not for visually guided saccades
( $5 \pm 3 \mathrm{~ms}$ increase, $p=0.20, z=1.29$, Wilcoxon signed-rank test). We surmise that the lack of a significant increase for visually guided saccades may relate both to the smaller accompanying changes in SRT with this task and to our stringent saccadematching analyses, as significant increases in accumulation onset were observed in the absence of saccade matching for both visually guided (increases of $16 \pm 3 \mathrm{~ms}, p<0.0001, z=4.82$, Wilcoxon signed-rank test) and memory-guided saccades (increases 
of $17 \pm 3 \mathrm{~ms}, p<0.0001, z=4.81$, Wilcoxon signed-rank test). More importantly, as shown in Figure 6C, increases or decreases in the onset of accumulation during FEF inactivation generally corresponded to a similar increase (e.g., closed circles clustering above the line of unity in Fig. 6C) or decrease (e.g., open circles clustering below the line of unity in Fig. $6 C)$ in SRT, respectively. To analyze this more closely, we plotted the change in the onset of accumulation versus the change in SRTs on a neuron-by-neuron basis, and determined the variance explained by a linear correlation (Fig. 6D). Interestingly, we found robust correlations across ipsilesional iSC neurons for both visually and memory-guided saccades $\left(r^{2}\right.$ values of 0.72 and $0.55, p<0.0001$ for each, $F$ statistics of 175.28 and 58.64 , respectively), with slopes near 1.0 (0.82 and 0.69, respectively) and intercepts near $0 \mathrm{~ms}$ ( 4 and $11 \mathrm{~ms}$, respectively). Notably, we also observed similar patterns in how changes in the onset of accumulation within the contralesional iSC predicted the accompanying changes in SRT $\left(r^{2}=0.44, p<\right.$ $0.01, F=12.83$ for visually guided saccades, and $r^{2}=0.60, p<0.001, F=23.68$ for memory-guided saccades). We verified our findings using various approaches to detect onset time (see Methods and Materials), including an alternative Poisson-based analysis. Results using this Poisson-based analysis agreed with the two-piece linearregression method, with FEF inactivation delaying the onset of accumulation in the ipsilesional iSC (increase of $6 \pm 4 \mathrm{~ms}, p=$ $0.13, z=1.51, n=71$ for visually guided saccades, and increase of $11 \pm 5 \mathrm{~ms}, p<0.0001, z=4.15, n=54$ for memory-guided saccades, Wilcoxon signed-rank tests), and the changes in onset remained strongly correlated with SRT differences $\left(r^{2}=0.53\right.$, $p<0.0001, F=78.41, m=0.53, y$-intercept $=6$ for visually guided saccades, and $r^{2}=0.64, p<0.0001, F=93.56, m=0.61$, $y$-intercept $=11$ for memory-guided saccades). Thus, on a neuron-by-neuron basis, changes in the onset of accumulation of saccade-related iSC activity predicted accompanying changes in SRT in an almost one-to-one manner.

FEF inactivation altered other parameters in the ipsilesional iSC neurons, but such changes did not predict accompanying SRT increases

SRT increases with FEF inactivation could also be related to increases in threshold activity or to decreases in baseline activity or rate of accumulation. While FEF inactivation did affect these parameters, the variability or direction of changes in neural activity did not relate as well to accompanying changes in SRT. For instance, FEF inactivation decreased the accumulation rate (4.6 \pm 1.1 to $3.5 \pm 1.1$ spikes $/ \mathrm{s}^{2}, p<0.0001, z=4.70$, Wilcoxon signedrank test $)$ and threshold activity $(210 \pm 40$ to $179 \pm 48$ spikes $/ \mathrm{s}$, $p<0.01, z=2.90$, Wilcoxon signed-rank test) in an exemplar neuron (Fig. 7A, arrow; same neuron as in Fig. $6 B$ ). Across our sample of ipsilesional iSC neurons, FEF inactivation consistently decreased baseline (Fig. $7 B$; decrease of $2 \pm 1$ spikes/s, $p<0.01$, $z=-2.60$, Wilcoxon signed-rank test) and threshold activity
(Fig. 7C; decrease of $11 \pm 3 \%, p<0.001, z=-3.34$, Wilcoxon signed-rank test) before memory-guided saccades, but not before visually guided saccades $(p=0.77$ and $0.13, z=-0.29$ and -1.52 , Wilcoxon signed-rank tests for baseline and threshold activity, respectively). FEF inactivation did not influence the rate of accumulation in either task (Fig. 7D; $p=0.76$ and $0.06, z=$ -0.30 and -1.85 , Wilcoxon signed-rank tests for visually and memory-guided tasks, respectively). Although changes in the rate of accumulation or baseline would be consistent with a neuronal mechanism that increases SRT during FEF inactivation, it is also important to consider interactions among parameters and how these collective changes could quantitatively relate to SRT increases.

To examine how well such parametric changes related to accompanying changes in SRT, we used a standard accumulator model that allowed us to extract the time-to-reach-threshold, computed from the measured values for baseline, threshold, and accumulation rate [(threshold - baseline activity)/(accumulation rate); see Materials and Methods]. Accumulator models are most commonly applied to speeded, rather than delayed, response tasks, but nevertheless such models provide a framework in which to relate changes in iSC activity to accompanying changes in SRT. Note that this time-to-reach-threshold value does not directly incorporate the onset of accumulation; effectively this model assumes that neural activity accumulates from baseline at some fixed time after a go-cue. The results of this analysis are shown on a neuron-by-neuron basis in Figure 7 E, F. While the time-to-reach-threshold in the ipsilesional iSC marginally increased with FEF inactivation for visually guided saccades $(1 \pm 1 \mathrm{~ms}$ increase, $p<0.05, z=2.25$, Wilcoxon signedrank test), we observed no consistent effects on memory-guided 

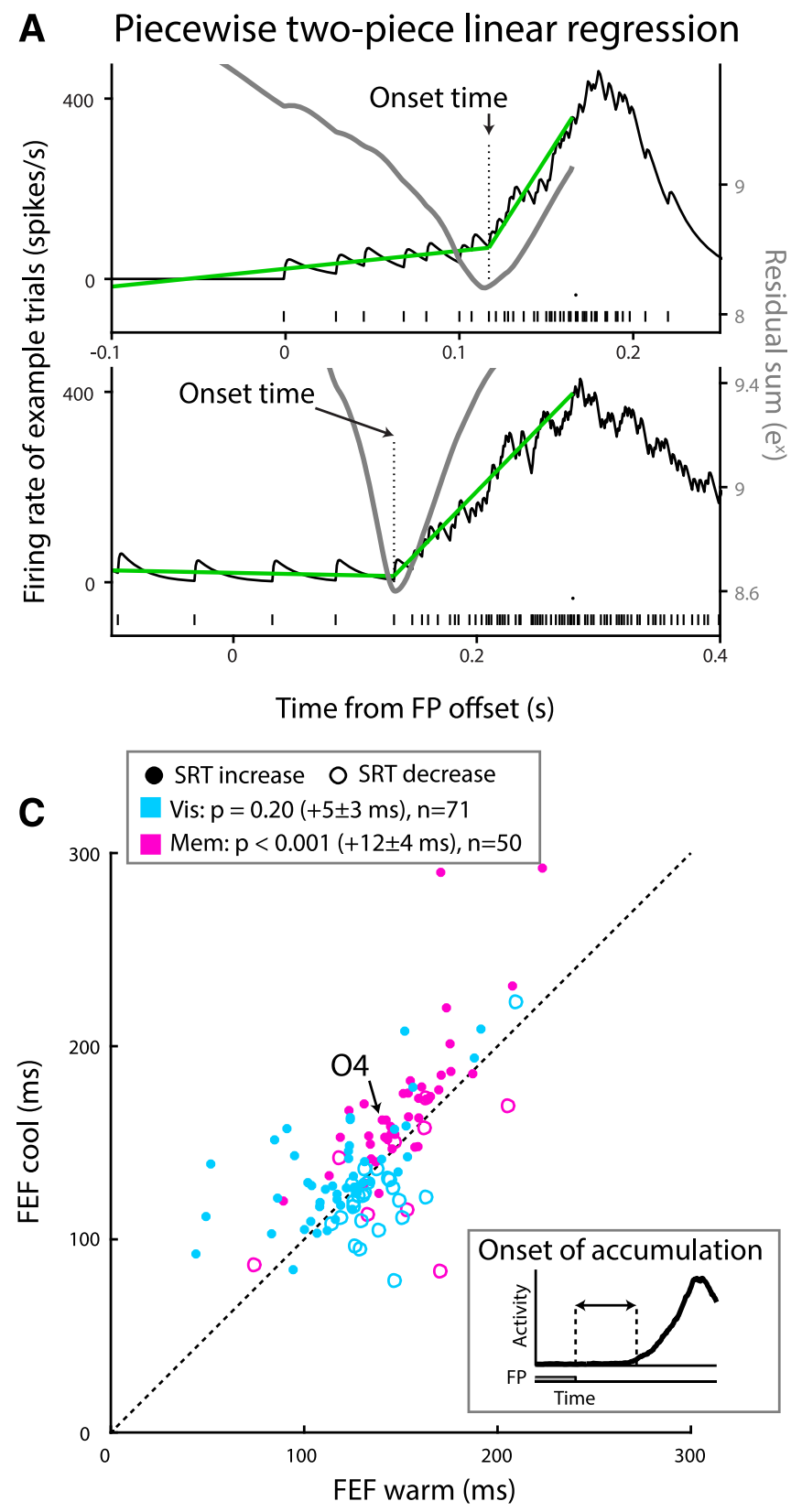

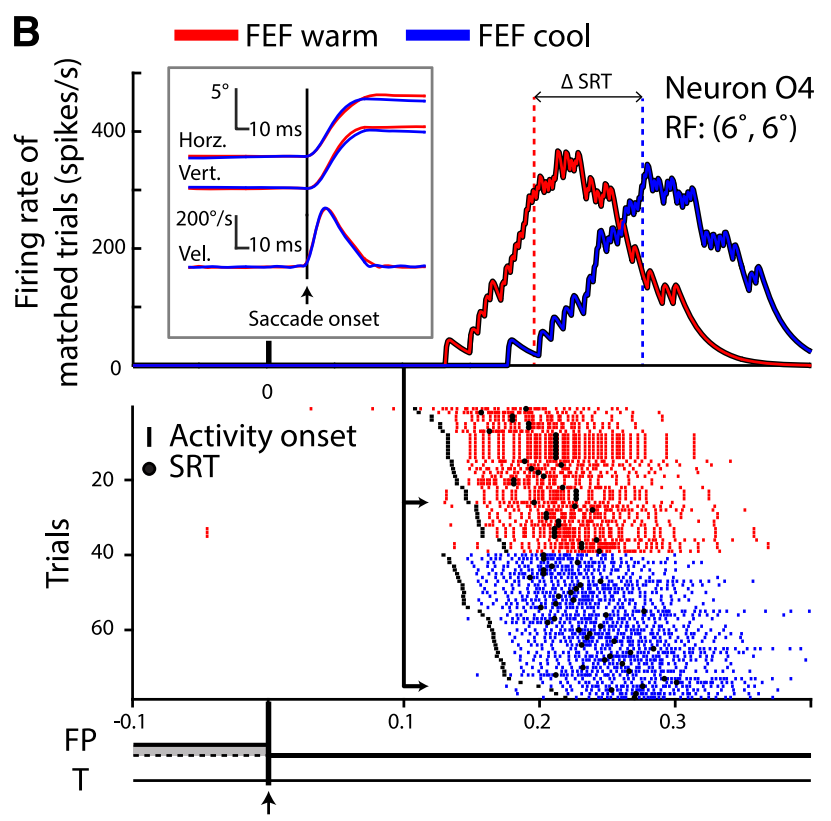

Time from FP offset (s)

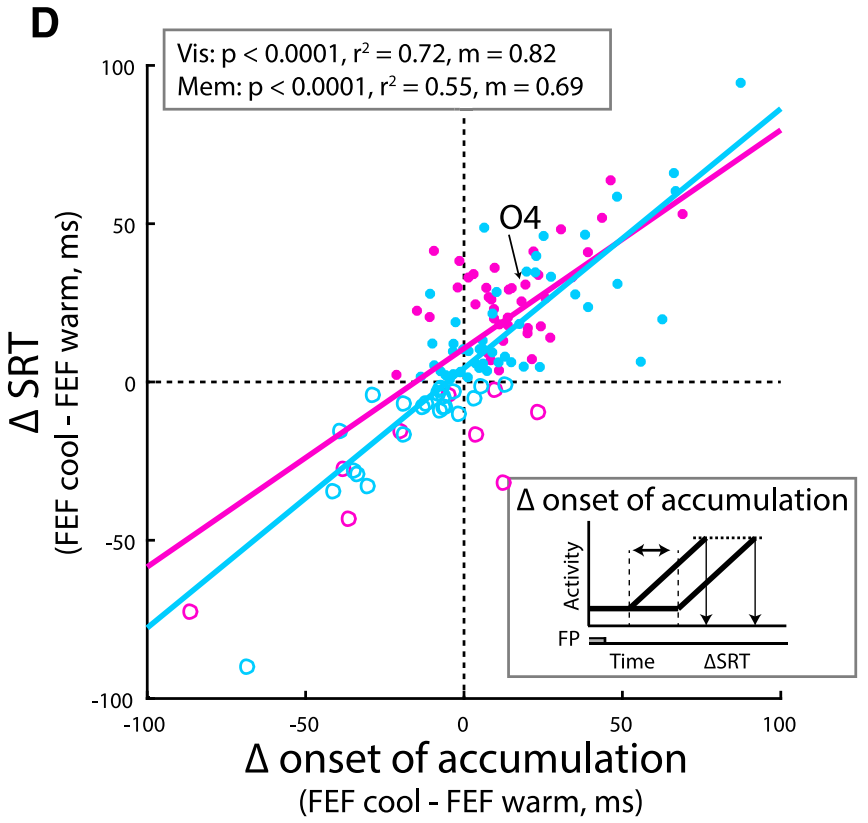

Figure 6. Inactivation-induced changes in SRT correlated with delays in the onset of saccade-related activity in the ipsilesional iSC. $\boldsymbol{A}$, Depiction of how onset time is detected using a piecewise two-piece linear-regression approach for two trials (see Materials and Methods). The onset time (dotted line) coincides with the inflection point that minimizes the summed squared error (gray curve, plotted against right axis) between convolved iSC activity (top, spike train shown below) and the two linear regressions (green lines). Note how the first linear regression captured any delay-period or build-up activity before the saccade (SRT is represented by the circle above raster plot). $\boldsymbol{B}$, For example ipsilesional neuron 04 , FEF inactivation delayed the onset of saccade-related activity (ticks) and SRT (circles) for matched memory-guided saccades. Top part shows spike-density function and kinematics (inset) of one matched pair; bottom part shows rasters for all matched saccades. $C$, FEF inactivation generally delayed the onset of saccade-related activity in ipsilesional iSC neurons. Each point shows the average change per neuron (across trials with matched saccades), with filled or open circles denoting SRT increases or decreases, respectively. $\boldsymbol{D}$, Changes in the onset of saccade-related activity strongly correlated with concomitant changes in SRT.

saccades ( $p=0.17, z=1.37$, Wilcoxon signed-rank test), and a linear regression of such changes against concomitant changes in SRT revealed no correlation in the ipsilesional iSC for either visually ( $p=0.35, F=0.89, r^{2}$ value of 0.01 ) or memory-guided saccades $\left(p=0.28, F=1.20, r^{2}\right.$ value of 0.02$)$. Moreover, any changes in the time-to-reach-threshold for the contralesional iSC did not relate at all to associated changes in ipsiversive SRT ( $p=$ 0.55 and $0.97, F=0.38$ and 0.001 for visually and memoryguided saccades, respectively). Thus, even when incorporating the three parameters of baseline, threshold, and accumulation rate, such changes do not fully account for the accompanying changes in SRT in the ipsilesional iSC.

Changes in the onset of accumulation best explained SRT differences across matched saccades, even without FEF inactivation

We extended the above analyses to the level of individually matched saccades, which enabled us to test how changes in onset 
A

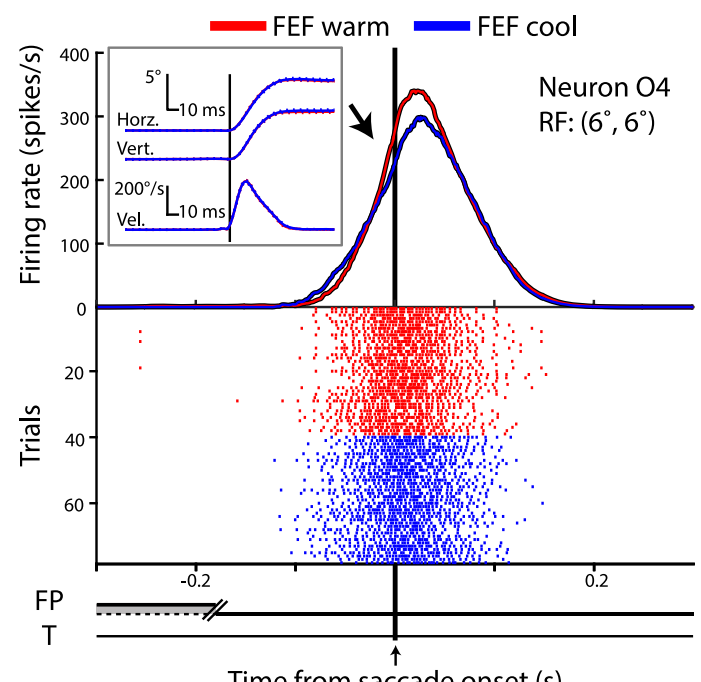

C

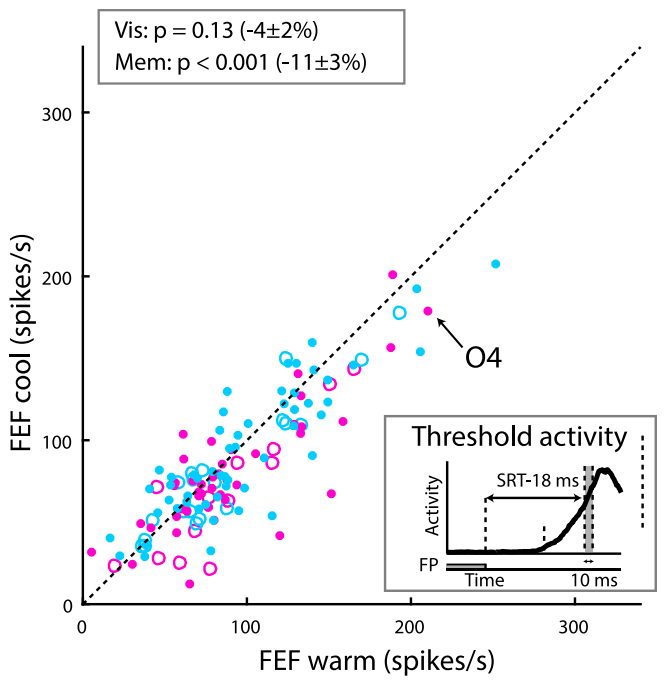

E

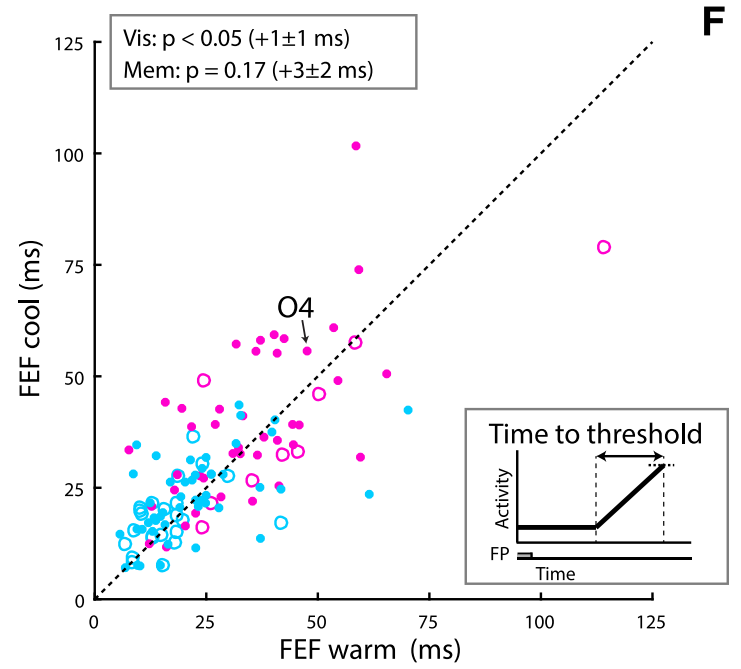

B

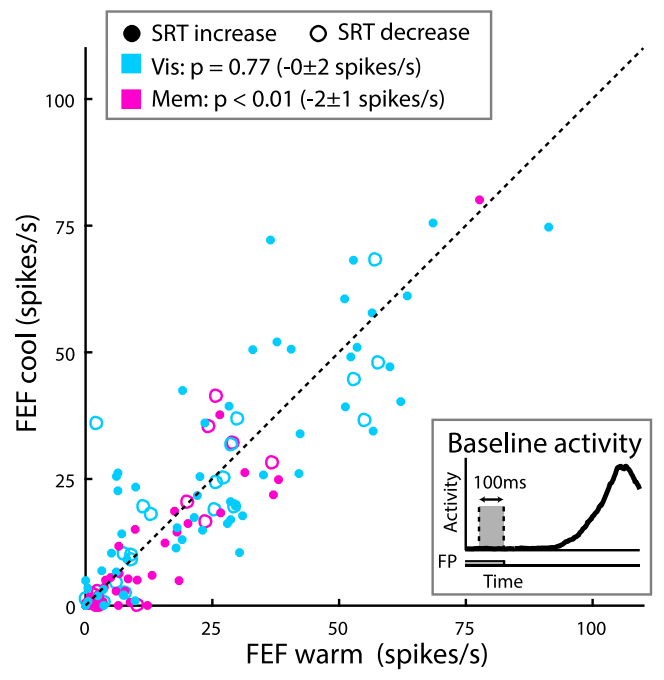

D

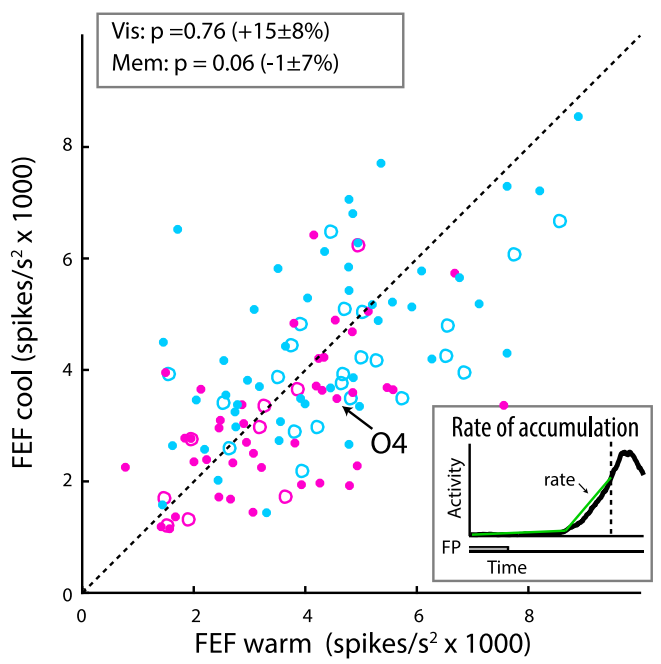

$F$

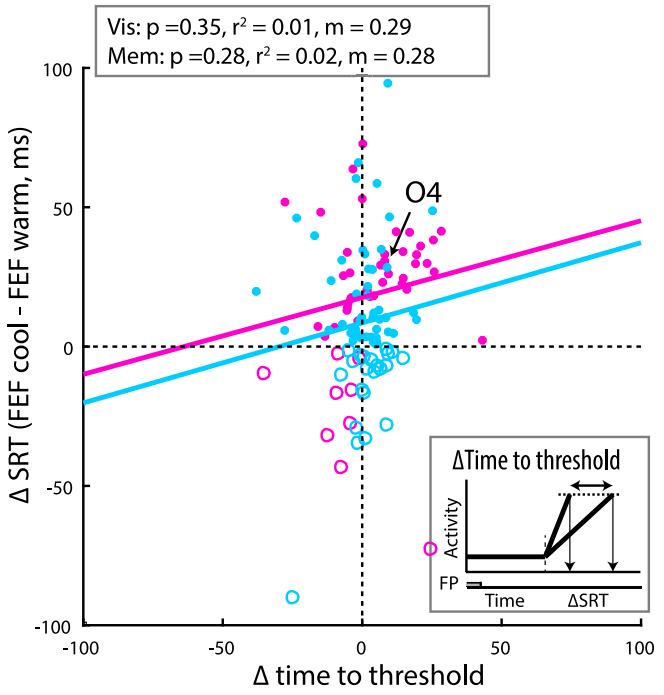

(FEF cool - FEF warm, ms)

Figure 7. FEF inactivation changed other aspects of iSC activity, but these changes poorly predicted changes in SRT. $A$, In example neuron 04 , FEF inactivation decreased both the accumulation rate and threshold activities (see Materials and Methods for how these parameters were measured) for matched memory-guided saccades. Same format as Figure $6 B$. $\boldsymbol{B}-\boldsymbol{D}$, Across our sample, FEF inactivation decreased baseline activity $(\boldsymbol{B})$, threshold activity $(\boldsymbol{C})$, and accumulation rate $(\boldsymbol{D})$ of ipsilesional isC neurons, particularly for memory-guided saccades. $\boldsymbol{E}$, $\boldsymbol{F}$, To analyze how changes in these parameters related to SRT differences in a one-to-one manner, we computed the time-to-reach-threshold as the difference of threshold and baseline activities divided by the accumulation rate. Note how this parameter does not directly incorporate the onset of accumulation, so it assumes that activity starts to accumulate at an arbitrary point in time after the go-cue. While such changes did increase the time-to-reach-threshold in the ipsilesional iSC $(\boldsymbol{E})$, such changes did not fully account for the concomitant changes in SRT $(\boldsymbol{F})$. 

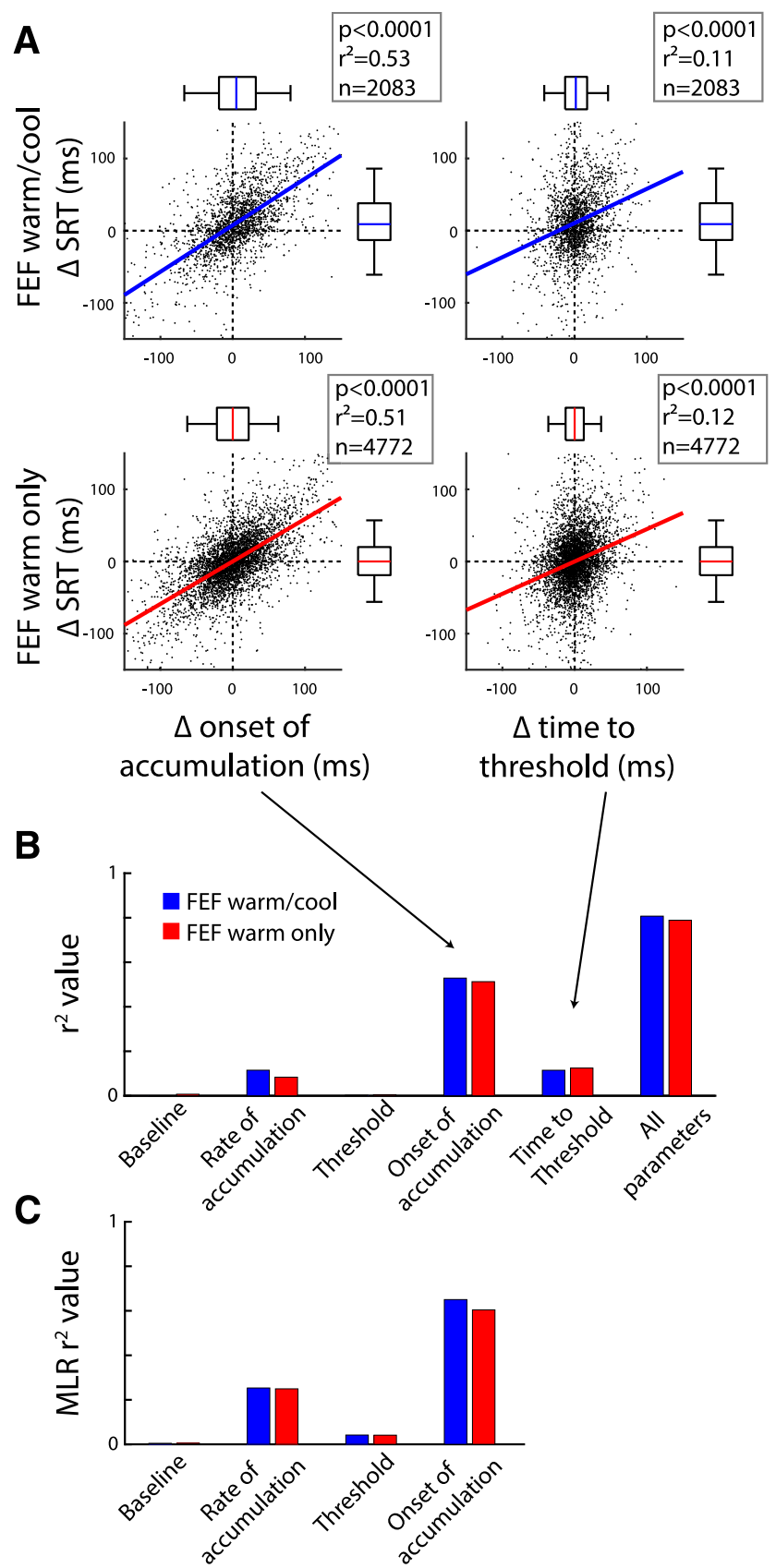

Figure 8. Across all matched trials, changes in the onset of accumulation best reflected changes in SRT, even without FEF inactivation. $A$, Across all matched trials extracted with (top row, blue lines) and without FEF inactivation (bottom row, red lines), differences in the onset of accumulation (left column) in the ipsilesional iSC related better to associated changes in SRT, compared with differences in the time-to-reach-threshold (right column). B, Amount of SRT variance explained by different combinations of individual or grouped parameters extracted from a rise-to-threshold model. Regardless of whether matched pairs were extracted across FEF inactivation or not, consideration of the change in the onset of accumulation greatly increased how well changes in iSC activity predicted concomitant changes in SRT. C, Across trials matched with or without FEF inactivation, the onset of accumulation best correlated with the remaining residual error following a multiple linear regression of the other individual parameters and SRT.

time correlated with a larger distribution of SRT changes. For each matched saccade pair, extracted either across FEF inactivation (Fig. $8 A$, top left subplot) or only from trials without FEF inactivation (Fig. $8 A$, bottom left subplot) from the ipsilesional iSC, we derived both a change in the onset of accumulation and a change in SRT. We pooled trials across saccade tasks, since we found largely equivalent results for visually or memory-guided saccades $\left(r^{2}\right.$ values of 0.62 and $0.42, F=1731.2$ and 622.8 , respectively, for relationship of changes in onset time vs SRT differences with FEF inactivation). Note that this procedure often matches trials where SRT decreased upon FEF inactivation, due to the overlap between response-time distributions when the FEF was or was not inactivated. Regardless, the changes in SRT and onset of accumulation remained highly correlated, regardless of whether the matched pairs were being compared across FEF inactivation or not ( $p$ 's $<0.0001, F=2201.4$ and $4684.1, r^{2}$ values of 0.53 and 0.51 , slopes of 0.65 and 0.59 , and $y$-intercepts of 8 and $0 \mathrm{~ms}$, respectively). In contrast, comparison of the change in the time-to-reach-threshold (computed from the baseline, rate of accumulation, and threshold) versus the change in SRT for matched-saccade pairs revealed much weaker relationships (Fig. $8 A$, right columns). Moreover, we found similarly poor relationships from a time-to-reach-threshold computed from a completely independent measure of accumulation rate $\left(r^{2}\right.$ values of 0.10 and 0.11 for with or without FEF inactivation, respectively; see Materials and Methods).

We repeated these analyses independently for each of the parameters of baseline, rate of accumulation, and threshold. As shown by $r^{2}$ values in Figure $8 B$, changes in these single parameters were also very poor predictors of accompanying changes in SRT. In contrast, consideration of the onset of accumulation either alone or in conjunction with all other parameters (i.e., adding the onset time of accumulation to the time-to-reachthreshold) greatly increased the relationship between changes in iSC activity and changes in SRT (Fig. $8 B$ ). Perhaps just as importantly, these observations held regardless of whether the matched pairs were compared across FEF inactivation or not (Fig. 8B, blue, red bars, respectively), or from contralesional iSC (data not shown). Comparable results were also obtained when we only matched saccades for metrics and not kinematics (data not shown).

Finally, to address how well a single parameter could independently explain SRT changes, even when allowing for correlation among other parameters, we adopted a multiple linear-regression approach (Hewitt et al., 2015) within the framework of a rise-tothreshold model of saccade initiation (see Materials and Methods). The objective of this analysis was to determine how much an individual parameter could independently explain the remaining variance following a multiple linear regression that correlates all the other parameters to SRT. As shown in Figure 8C, the onset of accumulation best accounted for the remaining variance from a multiple linear regression consisting of the other three parameters, reaffirming the importance of this parameter.

\section{Summary of results}

Reversible inactivation of a large volume of the unilateral FEF decreased all aspects of ipsilesional iSC activity, consistent with a general loss of excitatory input. The magnitude of such decreases in iSC activity depended both on the functional content of the signal, with greatest decreases for saccade-related activity, and on the inferred depth of the neuron within the SC, with greater decreases in visually related activity on those neurons also displaying delay-related and saccade-related activity. Such results largely conform both with the preferential distribution of frontal projections to intermediate and deeper layers of the iSC (Tigges and Tigges, 1981) and with antidromic studies of the functional content of corticotectal neurons (Segraves and Goldberg, 1987; Sommer and Wurtz, 2000; Helminski and Segraves, 2003). The absence of any effect of FEF inactivation on the latency of the first 
spike following visual-stimulus onset is also consistent with evidence that visual responses in the iSC require the integrity of the retinogeniculostriate pathway passing through the magnocellular laminae of the LGN (Schiller et al., 1979); perhaps not too surprisingly given the short response latencies, the FEF is also not a critical node in the pathway mediating the initial timing of the visual response in the iSC. Finally, by fitting the profiles of saccade-related iSC activity to a stochastic accumulator model, we showed that delays in the onset of saccade-related accumulation in either iSC, rather than systematic changes in either saccade threshold, baseline, or the rate of accumulation, best explained concomitant changes in SRT in the delayed saccade task, emphasizing the need to consider the onset of accumulation as a parameter in neurophysiologically inspired models of saccade initiation.

\section{Discussion}

FEF inactivation reduces excitatory input to the ipsilesional iSC without disinhibiting the contralesional iSC

The functional content of cortical signals relayed directly to iSC has been well characterized using antidromic identification (Segraves and Goldberg, 1987; Everling and Munoz, 2000; Sommer and Wurtz, 2000; Wurtz et al., 2001; Helminski and Segraves, 2003). However, due to other polysynaptic pathways, such as through the basal ganglia or other cortical areas, inactivation studies like ours are required to causally assess the collective influence of the FEF on iSC activity. The general agreement between our results and those that would have been predicted by antidromic studies alone is encouraging, reaffirming the FEF's role in providing excitatory input to the ipsilateral iSC, particularly for those neurons displaying saccade-related activity.

Long-range interactions between different regions of the iSC or between the FEFs are thought to be inhibitory (Munoz and Istvan, 1998; Schlag et al., 1998). Could the general reduction in ipsilesional iSC activity during FEF inactivation and the associated increases in response time arise from disinhibition of the contralesional FEF and/or iSC or from a shift toward increased activity of fixation neurons in the rostral iSC (Munoz and Wurtz, 1993; Dorris and Munoz, 1995)? While we did not record from the rostral iSC or contralesional FEF, several observations argue against these interpretations. First, the magnitude of contralesional iSC activity neither increased nor decreased, contrary to what would have been expected from disinhibition or increased rostral iSC activity, respectively. Second, unilateral FEF inactivation decreased the peak velocity and prevalence of cue-related microsaccades in both directions (Peel et al., 2016), which is more consistent with decreasing, rather than increasing, levels of rostral iSC activity (Hafed et al., 2009). Third, although FEF inactivation decreased the magnitude of visual responses on ipsilesional saccade-related iSC neurons, the latency of such responses was unchanged (Fig. 2). In contrast, during paradigms associated with increased rostral iSC activity, visual responses in the caudal iSC are both reduced in magnitude and delayed in onset latency (Marino et al., 2012).

Although FEF inactivation reduced ipsilesional iSC activity and delayed the onset of accumulation across our sample, there were instances where FEF inactivation had little to no effect on ipsilesional iSC activity, or decreased rather than increased the onset of accumulation. There are a number of potential reasons for this variability. It may be that not every iSC neuron we recorded receives input from the FEF, direct or otherwise. Further, any impact of FEF inactivation may be masked by compensatory inputs from other structures that converge on the same neuron.
To our knowledge, there is no data that speak to this level of anatomical and physiological detail within the oculomotor network. Additionally, given the topographically aligned nature of projections from the FEF to the iSC (Sommer and Wurtz, 2000), it is possible that we occasionally recorded from iSC locations unaffected by FEF inactivation. The question of spatial register may also explain why FEF inactivation occasionally decreased both SRTs and the onset of accumulation (Fig. $6 D$, points in the lower-left quadrant). We did not find any spatial dependency in how much FEF inactivation affected iSC activity or behavior (e.g., larger cooling effects did not stem from particular parts of the visual field), but this analysis was hampered by the variability in loop location across monkeys and hemispheres.

\section{A bilateral influence of the unilateral FEF on SRT and the onset of saccade-related accumulation in the iSC}

The failure to observe disinhibition in the contralesional iSC is surprising given that focal pharmacological inactivation of the FEF facilitates ipsiversive oculomotor behaviors (Sommer and Tehovnik, 1997; Dias and Segraves, 1999; Wardak et al., 2006). Studies using focal microstimulation (Schlag et al., 1998; Seidemann et al., 2002) or paired bilateral recordings (Cohen et al., 2010) have also supported a view wherein the two FEFs compete in a push-pull fashion. In contrast, large-volume FEF inactivation tends to delay rather than facilitate ipsiversive oculomotor behaviors (Fig. 1; Peel et al., 2014, 2016; Kunimatsu et al., 2015), and although ipsiversive SRT increases were of lower magnitude and more idiosyncratic than the increases in contraversive SRTs, when present such SRT increases related best to delays in the onset of saccade-related accumulation in the corresponding iSC.

The behavioral and neurophysiological results produced by unilateral cryogenic inactivation of the FEF, reported here and elsewhere (Peel et al., 2014, 2016), also differ from that seen following unilateral cryogenic inactivation of the adjacent dorsolateral prefrontal cortex (DLPFC). As reported by Koval et al. (2011) and Johnston et al. (2014), unilateral cryogenic inactivation of the DLPFC via cooling loops implanted within the caudal principal sulcus shortens the response times of ipsiversive saccades and increases preparatory, visual-related, and saccade-related activity in the contralesional iSC. Thus, unlike what we observed following unilateral inactivation of the FEF, unilateral cryogenic inactivation of the nearby DLPFC does produce results consistent with disinhibition via a push-pull mechanism. Importantly, since the cooling loops implanted in the FEF or DLPFC were placed within the arcuate or caudal principal sulci respectively, it is unlikely that they inactivated overlapping volumes of tissue on the gyral crowns.

\section{New perspectives on saccade initiation}

FEF inactivation altered how iSC activity relates to saccade initiation in a manner that provides new insights into how the oculomotor brainstem initiates a saccade. One fundamental observation is that less saccade-related activity is emitted by ipsilateral iSC neurons during FEF inactivation, even for saccades matched closely for metrics and kinematics. Clearly, at the level of single iSC neurons, the saccade threshold can vary for movements to the center of the response field (Fig. 7). This observation complements observations of changes in the saccade threshold in the iSC (Everling et al., 1999; Jantz et al., 2013) or FEF (Heitz and Schall, 2012) during different tasks or cognitive sets. Heitz and Schall (2012) reconciled observations of increased SRTs despite decreasing thresholds by proposing a leaky integrator mechanism where saccade-related spikes 
are integrated over time to produce an invariant level of cumulative activity, and our results of decreasing thresholds and rates of accumulation upon FEF inactivation show that a similar mechanism may apply to the iSC. The decreased activation of iSC neurons for movements to the center of the response field could also be offset by increased activity from other off-center iSC neurons, extending the notion of saccade threshold within the iSC to forms of population coding envisaged for other aspects of saccade control (for review, see Gandhi and Katnani, 2011). It is also possible that an overall decrease in iSC activity could be offset by increasing activity in other areas that project to the brainstem burst generator, such as the fastigial nucleus (Noda et al., 1990), to produce an overall equivalent input to the brainstem burst generator. Understanding the contribution of these other cell populations, either within or outside of the iSC, is presumably required to fully explain SRT changes with FEF inactivation.

Previous evidence for fixed thresholds in the FEF (Hanes and Schall, 1996; Brown et al., 2008) and iSC (Paré and Hanes, 2003) fit well with rise-to-threshold (Carpenter and Williams, 1995; Reddi and Carpenter, 2000; Lo and Wang, 2006; Carpenter et al., 2009) and drift-diffusion models (Ratcliff et al., 2003, 2007) of saccade initiation, strengthening contentions that these models provided useful descriptions of neural activity. However, by embracing a greater subset of experimental tasks or conditions, the work of Heitz and Schall $(2012,2013)$ and Jantz and colleagues (2013) revealed that contemporary stochastic accumulator models failed to predict observed profiles of FEF or iSC activity. More broadly, delays in the onset of movement-related activity in the FEF and/or iSC, rather than changes in threshold or rate of accumulation, relate best to SRT increases in more difficult visual-search paradigms (Woodman et al., 2008), or to post-error increases in SRT within a stop-signal paradigm (Pouget et al., 2011). A recent review has emphasized the importance of incorporating the onset of activity within studies of decision making, particularly in tasks requiring top-down regulation (Teichert et al., 2016). Our inactivation findings, as well as our comparison of matched saccades even without FEF inactivation, extend these findings by demonstrating the importance of the onset of saccade-related activity in the iSC in delayed-response tasks, and by showing that such onset is governed at least in part by inputs from the FEF. It is becoming increasingly clear that the onset of saccade-related activity is a relevant metric that affects SRT. Future studies incorporating FEF inactivation and iSC recording during the performance of more unconstrained tasks requiring either a speeded response or target selection and decision making in light of multiple competing options are necessary to see whether our results generalize more widely; it may well be that variations in accumulation rate are critical determinants of SRT in such tasks. The results in the current report will serve as a benchmark for such efforts.

\section{References}

Anderson RW, Keller EL, Gandhi NJ, Das S (1998) Two-dimensional saccade-related population activity in superior colliculus in monkey. J Neurophysiol 80:798-817. Medline

Basso MA, Wurtz RH (1998) Modulation of neuronal activity in superior colliculus by changes in target probability. J Neurosci 18:7519-7534. Medline

Berman RA, Joiner WM, Cavanaugh J, Wurtz RH (2009) Modulation of presaccadic activity in the frontal eye field by the superior colliculus. J Neurophysiol 101:2934-2942. CrossRef Medline

Brown JW, Hanes DP, Schall JD, Stuphorn V (2008) Relation of frontal eye field activity to saccade initiation during a countermanding task. Exp Brain Res 190:135-151. CrossRef Medline

Carpenter RH, Williams ML (1995) Neural computation of log likelihood in control of saccadic eye movements. Nature 377:59-62. CrossRef Medline
Carpenter RH, Reddi BA, Anderson AJ (2009) A simple two-stage model predicts response time distributions. J Physiol 587:4051-4062. CrossRef Medline

Cashaback JG, Cluff T, Potvin JR (2013) Muscle fatigue and contraction intensity modulates the complexity of surface electromyography. J Electromyogr Kinesiol 23:78-83. CrossRef Medline

Cohen JY, Crowder EA, Heitz RP, Subraveti CR, Thompson KG, Woodman GF, Schall JD (2010) Cooperation and competition among frontal eye field neurons during visual target selection. J Neurosci 30:3227-3238. CrossRef Medline

Crapse TB, Sommer MA (2009) Frontal eye field neurons with spatial representations predicted by their subcortical input. J Neurosci 29:53085318. CrossRef Medline

Dias EC, Segraves MA (1999) Muscimol-induced inactivation of monkey frontal eye field: effects on visually and memory-guided saccades. J Neurophysiol 81:2191-2214. Medline

Ding L, Gold JI (2012) Neural correlates of perceptual decision making before, during, and after decision commitment in monkey frontal eye field. Cereb Cortex 22:1052-1067. CrossRef Medline

Dorris MC, Munoz DP (1995) A neural correlate for the gap effect on saccadic reaction times in monkey. J Neurophysiol 73:2558-2562. Medline

Dorris MC, Paré M, Munoz DP (1997) Neuronal activity in monkey superior colliculus related to the initiation of saccadic eye movements. J Neurosci 17:8566-8579. Medline

Everling S, Munoz DP (2000) Neuronal correlates for preparatory set associated with pro-saccades and anti-saccades in the primate frontal eye field. J Neurosci 20:387-400. Medline

Everling S, Dorris MC, Klein RM, Munoz DP (1999) Role of primate superior colliculus in preparation and execution of anti-saccades and prosaccades. J Neurosci 19:2740-2754. Medline

Gandhi NJ, Katnani HA (2011) Motor functions of the superior colliculus. Annu Rev Neurosci 34:205-231. CrossRef Medline

Goonetilleke SC, Katz L, Wood DK, Gu C, Huk AC, Corneil BD (2015) Cross-species comparison of anticipatory and stimulus-driven neck muscle activity well before saccadic gaze shifts in humans and nonhuman primates. J Neurophysiol 114:902-913. CrossRef Medline

Hafed ZM, Chen CY (2016) Sharper, stronger, faster upper visual field representation in primate superior colliculus. Curr Biol 26:1647-1658. CrossRef Medline

Hafed ZM, Goffart L, Krauzlis RJ (2009) A neural mechanism for microsaccade generation in the primate superior colliculus. Science 323:940-943. CrossRef Medline

Hanes DP, Schall JD (1996) Neural control of voluntary movement initiation. Science 274:427-430. CrossRef Medline

Hanes DP, Thompson KG, Schall JD (1995) Relationship of presaccadic activity in frontal eye field and supplementary eye field to saccade initiation in macaque: Poisson spike train analysis. Exp Brain Res 103:85-96. Medline

Heitz RP, Schall JD (2012) Neural mechanisms of speed-accuracy tradeoff. Neuron 76:616-628. CrossRef Medline

Heitz RP, Schall JD (2013) Neural chronometry and coherency across speed-accuracy demands reveal lack of homomorphism between computational and neural mechanisms of evidence accumulation. Philos Trans R Soc Lond B Biol Sci 368:20130071. CrossRef Medline

Helminski JO, Segraves MA (2003) Macaque frontal eye field input to saccade-related neurons in the superior colliculus. J Neurophysiol 90: 1046-1062. CrossRef Medline

Hewitt AL, Popa LS, Ebner TJ (2015) Changes in Purkinje cell simple spike encoding of reach kinematics during adaption to a mechanical perturbation. J Neurosci 35:1106-1124. CrossRef Medline

Huerta MF, Krubitzer LA, Kaas JH (1986) Frontal eye field as defined by intracortical microstimulation in squirrel monkeys, owl monkeys, and macaque monkeys: I. Subcortical connections. J Comp Neurol 253:415439. CrossRef Medline

Jantz JJ, Watanabe M, Everling S, Munoz DP (2013) Threshold mechanism for saccade initiation in frontal eye field and superior colliculus. J Neurophysiol 109:2767-2780. CrossRef Medline

Johnston K, Koval MJ, Lomber SG, Everling S (2014) Macaque dorsolateral prefrontal cortex does not suppress saccade-related activity in the superior colliculus. Cereb Cortex 24:1373-1388. CrossRef Medline

Katnani HA, Gandhi NJ (2012) The relative impact of microstimulation 
parameters on movement generation. J Neurophysiol 108:528-538. CrossRef Medline

Komatsu H, Suzuki H (1985) Projections from the functional subdivisions of the frontal eye field to the superior colliculus in the monkey. Brain Res 327:324-327. CrossRef Medline

Koval MJ, Lomber SG, Everling S (2011) Prefrontal cortex deactivation in macaques alters activity in the superior colliculus and impairs voluntary control of saccades. J Neurosci 31:8659-8668. CrossRef Medline

Kunimatsu J, Miyamoto N, Ishikawa M, Shirato H, Tanaka M (2015) Application of radiosurgical techniques to produce a primate model of brain lesions. Front Syst Neurosci 9:67. CrossRef Medline

Leichnetz GR, Spencer RF, Hardy SG, Astruc J (1981) The prefrontal corticotectal projection in the monkey: an anterograde and retrograde horseradish peroxidase study. Neuroscience 6:1023-1041. CrossRef Medline

Lo CC, Wang XJ (2006) Cortico-basal ganglia circuit mechanism for a decision threshold in reaction time tasks. Nat Neurosci 9:956-963. CrossRef Medline

Lomber SG, Payne BR, Horel JA (1999) The cryoloop: an adaptable reversible cooling deactivation method for behavioral or electrophysiological assessment of neural function. J Neurosci Methods 86:179-194. CrossRef Medline

Marino RA, Levy R, Boehnke S, White BJ, Itti L, Munoz DP (2012) Linking visual response properties in the superior colliculus to saccade behavior. Eur J Neurosci 35:1738-1752. CrossRef Medline

McPeek RM, Keller EL (2002) Saccade target selection in the superior colliculus during a visual search task. J Neurophysiol 88:2019-2034. Medline

Miyashita N, Hikosaka O (1996) Minimal synaptic delay in the saccadic output pathway of the superior colliculus studied in awake monkey. Exp Brain Res 112:187-196. Medline

Munoz DP, Istvan PJ (1998) Lateral inhibitory interactions in the intermediate layers of the monkey superior colliculus. J Neurophysiol 79:11931209. Medline

Munoz DP, Wurtz RH (1993) Fixation cells in monkey superior colliculus. I. Characteristics of cell discharge. J Neurophysiol 70:559-575. Medline

Munoz DP, Wurtz RH (1995) Saccade-related activity in monkey superior colliculus. I. Characteristics of burst and buildup cells. J Neurophysiol 73:2313-2333. Medline

Nagy B, Corneil BD (2010) Representation of horizontal head-on-body position in the primate superior colliculus. J Neurophysiol 103:858-874. CrossRef Medline

Noda H, Sugita S, Ikeda Y (1990) Afferent and efferent connections of the oculomotor region of the fastigial nucleus in the macaque monkey. J Comp Neurol 302:330-348. CrossRef Medline

Pandya DN, Vignolo LA (1971) Intra- and interhemispheric projections of the precentral, premotor and arcuate areas in the rhesus monkey. Brain Res 26:217-233. CrossRef Medline

Paré M, Hanes DP (2003) Controlled movement processing: superior colliculus activity associated with countermanded saccades. J Neurosci 23: 6480-6489. Medline

Peel TR, Johnston K, Lomber SG, Corneil BD (2014) Bilateral saccadic deficits following large and reversible inactivation of unilateral frontal eye field. J Neurophysiol 111:415-433. CrossRef Medline

Peel TR, Hafed ZM, Dash S, Lomber SG, Corneil BD (2016) A causal role for the cortical frontal eye fields in microsaccade deployment. PLoS Biol 14:e1002531. CrossRef Medline

Pouget P, Logan GD, Palmeri TJ, Boucher L, Paré M, Schall JD (2011) Neural basis of adaptive response time adjustment during saccade countermanding. J Neurosci 31:12604-12612. CrossRef Medline

Ratcliff R, Cherian A, Segraves M (2003) A comparison of macaque behavior and superior colliculus neuronal activity to predictions from models of two-choice decisions. J Neurophysiol 90:1392-1407. CrossRef Medline

Ratcliff R, Hasegawa YT, Hasegawa RP, Smith PL, Segraves MA (2007) Dual diffusion model for single-cell recording data from the superior col- liculus in a brightness-discrimination task. J Neurophysiol 97:1756-1774. Medline

Raybourn MS, Keller EL (1977) Colliculoreticular organization in primate oculomotor system. J Neurophysiol 40:861-878. Medline

Reddi BA, Carpenter RH (2000) The influence of urgency on decision time. Nat Neurosci 3:827-830. CrossRef Medline

Rezvani S, Corneil BD (2008) Recruitment of a head-turning synergy by low-frequency activity in the primate superior colliculus. J Neurophysiol 100:397-411. CrossRef Medline

Schall JD (2015) Visuomotor functions in the frontal lobe. Annu Rev Vis Sci 1:469-498. CrossRef Medline

Schiller PH, Malpeli JG, Schein SJ (1979) Composition of geniculostriate input of superior colliculus of the rhesus monkey. J Neurophysiol 42: 1124-1133. Medline

Schlag J, Dassonville P, Schlag-Rey M (1998) Interaction of the two frontal eye fields before saccade onset. J Neurophysiol 79:64-72. Medline

Schnyder H, Reisine H, Hepp K, Henn V (1985) Frontal eye field projection to the paramedian pontine reticular formation traced with wheat germ agglutinin in the monkey. Brain Res 329:151-160. CrossRef Medline

Segraves MA, Goldberg ME (1987) Functional properties of corticotectal neurons in the monkey's frontal eye field. J Neurophysiol 58:1387-1419. Medline

Seidemann E, Arieli A, Grinvald A, Slovin H (2002) Dynamics of depolarization and hyperpolarization in the frontal cortex and saccade goal. Science 295:862-865. CrossRef Medline

Shankar S, Massoglia DP, Zhu D, Costello MG, Stanford TR, Salinas E (2011) Tracking the temporal evolution of a perceptual judgment using a compelled-response task. J Neurosci 31:8406-8421. CrossRef Medline

Sommer MA, Tehovnik EJ (1997) Reversible inactivation of macaque frontal eye field. Exp Brain Res 116:229-249. CrossRef Medline

Sommer MA, Wurtz RH (2000) Composition and topographic organization of signals sent from the frontal eye field to the superior colliculus. J Neurophysiol 83:1979-2001. Medline

Sommer MA, Wurtz RH (2004) What the brain stem tells the frontal cortex. I. Oculomotor signals sent from superior colliculus to frontal eye field via mediodorsal thalamus. J Neurophysiol 91:1381-1402. CrossRef Medline

Stanford TR, Freedman EG, Sparks DL (1996) Site and parameters of microstimulation: evidence for independent effects on the properties of saccades evoked from the primate superior colliculus. J Neurophysiol 76: 3360-3381. Medline

Teichert T, Grinband J, Ferrera V (2016) The importance of decision onset. J Neurophysiol 115:643-661. CrossRef Medline

Thompson KG, Hanes DP, Bichot NP, Schall JD (1996) Perceptual and motor processing stages identified in the activity of macaque frontal eye field neurons during visual search. J Neurophysiol 76:4040-4055. Medline

Tigges J, Tigges M (1981) Distribution of retinofugal and corticofugal axon terminals in the superior colliculus of squirrel monkey. Invest Ophthalmol Vis Sci 20:149-158. Medline

Waitzman DM, Ma TP, Optican LM, Wurtz RH (1991) Superior colliculus neurons mediate the dynamic characteristics of saccades. J Neurophysiol 66:1716-1737. Medline

Wardak C, Ibos G, Duhamel JR, Olivier E (2006) Contribution of the monkey frontal eye field to covert visual attention. J Neurosci 26:4228-4235. CrossRef Medline

Woodman GF, Kang MS, Thompson K, Schall JD (2008) The effect of visual search efficiency on response preparation: neurophysiological evidence for discrete flow. Psychol Sci 19:128-136. CrossRef Medline

Wurtz RH, Sommer MA, Paré M, Ferraina S (2001) Signal transformations from cerebral cortex to superior colliculus for the generation of saccades. Vision Res 41:3399-3412. CrossRef Medline

Yoshida K, Iwamoto Y, Chimoto S, Shimazu H (1999) Saccade-related inhibitory input to pontine omnipause neurons: an intracellular study in alert cats. J Neurophysiol 82:1198-1208. Medline 